Draft Version June 20, 2018

Preprint typeset using $\mathrm{LAT}_{\mathrm{E} X} \mathrm{X}$ style emulateapj v. 06/18/13

\title{
THE DEPENDENCE OF C IV BROAD ABSORPTION LINE PROPERTIES ON ACCOMPANYING SI IV AND AL III ABSORPTION: RELATING QUASAR-WIND IONIZATION LEVELS, KINEMATICS, AND COLUMN DENSITIES
}

\author{
N. Filiz AK ${ }^{1,2,3}$, W. N. Brandt ${ }^{1,2}$, P. B. Hall ${ }^{4}$, D. P. Schneider ${ }^{1,2}$, J. R. Trump ${ }^{1,2,5}$, S. F. Anderson ${ }^{6}$, F. Hamann $^{7}$, \\ Adam D. Myers ${ }^{8}$, I. PÂris ${ }^{9}$, P. Petitjean ${ }^{10}$, Nicholas P. Ross ${ }^{11,12}$, Yue Shen ${ }^{5,13}$, Don York ${ }^{14,15}$ \\ Draft version June 20, 2018
}

\begin{abstract}
We consider how the profile and multi-year variability properties of a large sample of C IV Broad Absorption Line (BAL) troughs change when BALs from Si IV and/or Al III are present at corresponding velocities, indicating that the line-of-sight intercepts at least some lower ionization gas. We derive a number of observational results for CIV BALs separated according to the presence or absence of accompanying lower ionization transitions, including measurements of composite profile shapes, equivalent width $(\mathrm{EW})$, characteristic velocities, composite variation profiles, and EW variability. We also measure the correlations between EW and fractional-EW variability for C IV, Si IV, and Al III. Our measurements reveal the basic correlated changes between ionization level, kinematics, and column density expected in accretion-disk wind models; e.g., lines-of-sight including lower ionization material generally show deeper and broader C IV troughs that have smaller minimum velocities and that are less variable. Many C IV BALs with no accompanying Si IV or Al III BALs may have only mild or no saturation.
\end{abstract}

\section{INTRODUCTION}

Broad Absorption Lines (BALs) in quasar spectra are seen as a result of high-velocity outflows. The most commonly used empirical definition of BALs requires an absorption feature to have at least a $2000 \mathrm{~km} \mathrm{~s}^{-1}$ width at $10 \%$ under the continuum level (e.g., Wevmann et al. 1991). BAL quasars exhibit such broad absorption troughs in a wide variety of species in their rest-frame ultraviolet spectra, such as $\mathrm{P}_{\mathrm{V}} \lambda \lambda 1118,1128 \AA$, Ly $\alpha$ $\lambda 1216 \AA$, N v $\lambda \lambda 1239,1243 \AA$, Si IV $\lambda \lambda 1394,1403 \AA$, C IV $\lambda \lambda 1548,1551 \AA$, Al II $\lambda 1671 \AA$, Al III $\lambda \lambda 1855,1863 \AA$, and Mg II $\lambda \lambda 2797,2804 \AA$.

nfilizak@astro.psu.edu

${ }^{1}$ Department of Astronomy \& Astrophysics, Pennsylvania State University, University Park, PA, 16802, USA

2 Institute for Gravitation and the Cosmos, Pennsylvania State University, University Park, PA 16802, USA

${ }^{3}$ Faculty of Sciences, Department of Astronomy and Space Sciences, Erciyes University, 38039 Kayseri, Turkey

${ }^{4}$ Department of Physics and Astronomy, York University, 4700 Keele St., Toronto, Ontario, M3J 1P3, Canada

5 Hubble Fellow

6 Astronomy Department, University of Washington, Seattle, WA 98195, USA

7 Department of Astronomy, University of Florida, Gainesville, FL 32611-2055, USA

${ }^{8}$ Department of Physics and Astronomy, University of Wyoming, Laramie, WY 82071, USA

9 Departamento de Astronomía, Universidad de Chile, Casilla 36-D, Santiago, Chile

10 Universite Paris 6, Institut d'Astrophysique de Paris, 75014, Paris, France

11 Lawrence Berkeley National Laboratory, 1 Cyclotron Road, Berkeley, CA 94720, USA

12 Department of Physics, Drexel University, 3141 Chestnut Street, Philadelphia, PA 19104, USA

13 Carnegie Observatories, 813 Santa Barbara Street, Pasadena, CA 91101, USA

14 The University of Chicago, Department of Astronomy and Astrophysics, Chicago, IL 60637, USA

15 The University of Chicago, Enrico Fermi Institute, Chicago, IL 60637, USA
BAL quasars are classified into three groups based on the observed transitions in their spectra. The majority of BAL quasars exhibit absorption from only highionization transitions such as Nv, Si IV, and C IV (HiBALs, e.g., Weymann et al. 1991). Approximately 10\% of BAL quasars in optically selected samples also exhibit absorption from low-ionization transitions such as Al II, $\mathrm{Al}$ III, and Mg II (LoBALs, e.g., Voit et al. 1993; Gibson et al. 2009). Only $\approx 1 \%$ of BAL quasars show absorption from excited states of Fe II and/or Fe III in addition to the high and low-ionization transitions listed above (FeLoBALs, e.g., Becker et al. 2000; Hall et al. 2002). The existence of these groups indicates that quasar outflows can have a wide range of ionization states. It has been argued that the presence of the lowionization lines is not the only difference between these groups (e.g., Boroson \& Meyers 1992; Turnshek et al. 1994; Zhang et al. 2010). The generally weak [O III] emission and strong reddening of LoBALs suggest that LoBALs tend to be surrounded by dust and gas that has a larger global covering factor compared to HiBALs.

The details of the structure and geometry of quasar outflows remain unclear. A commonly adopted and welldeveloped model suggests that many BALs are formed in an equatorial wind that is launched from the accretion disk at $\approx 10^{16}-10^{17} \mathrm{~cm}$ from the central supermassive black hole (SMBH) for black-hole masses of $10^{8}-10^{9} \mathrm{M}_{\odot}$ and is driven by radiation pressure (e.g., Murray et al. 1995; Proga et al. 2000; Higginbottom et al. 2013). This disk-wind model successfully explains several important observational facts about BAL quasars, such as the presence of absorption from both high and low-ionization transitions despite the luminous ionizing radiation from the central source, and the large range of outflow velocities that reach from the systemic velocity up to $0.1 \mathrm{c}$. Numerical hydrodynamical simulations of the disk-wind model provide detailed predictions of the structure and dynamics of quasar outflows (e.g., Proga et al. 2000). 
Previous studies have shown that some BAL troughs are much more optically thick than they appear and that the depths of such troughs only mildly depend on column density. Several pieces of evidence for this line-saturation interpretation have been presented, such as $\mathrm{P}_{\mathrm{V}}$ BALs, depth differences in unblended doublet lines, and "flat-bottom" BAL profiles (e.g., Arav 1997; Hamann 1998; Arav et al. 1999a, b, 2001; Leighly et al. 2009; Borguet et al. 2012). For instance, Hamann (1998) studied spectra of the BAL quasar PG $1254+047$ which possesses relatively strong $\mathrm{P}_{\mathrm{V}}$ absorption at velocities corresponding to non-black strong $\mathrm{C}$ IV and SIV BAL troughs. The existence of $\mathrm{P} \mathrm{V}$ absorption was taken as evidence for saturated C IV absorption since phosphorus is expected to be $\sim 1000$ times less abundant than carbon (based on solar abundances). As another example, Arav et al. (1999b) argued that the depth differences between the unblended Si IV doublet lines of the quasar FIRST J1603+3002 arise as a result of velocitydependent partial coverage. Calculating the optical depths, they found that the CIV and Si IV absorption lines are saturated. Such studies have suggested that the non-black nature of these saturated lines arises due to partial coverage of the emission source along the lineof-sight; BAL troughs do not reach zero intensity due to photons from the emission source that are not absorbed and/or are scattered into the observer's line-ofsight. Supporting this argument, spectropolarimetric observations of BAL quasars have shown that the fractional contribution from scattered emission often increases at the wavelengths where BAL troughs are found, indicating an excess of scattered light relative to direct light (e.g., Ogle et al. 1999). These lines of observational evidence indicate that some BAL quasars have highly saturated BAL troughs and that the depths of such troughs are largely set by the line-of-sight covering factor rather than column density. However, detailed studies of line saturation are only available for a limited number of objects and have often focused on deep C IV BALs. It is possible that some BALs might be weak simply because the column density along the line-of-sight is small.

Recent sample-based investigations of BAL variability have brought new insights about the structure, dynamics, and evolution of quasar outflows showing that variability is common for most BAL troughs on multiyear timescales (e.g., Lundgren et al. 2007; Gibson et al. 2008, 2010; Capellupo et al. 2011, 2012; Filiz Ak et al. 2012, 2013; Vivek et al. 2012; Wildy et al. 2013). These studies have revealed that the fractional variation of BAL troughs in lower ionization transitions (such as Si IV) is generally stronger than that in C IV (e.g., Capellupo et al. 2012; Vivek et al. 2012; Filiz Ak et al. 2013). A recent study by Filiz Ak et al. (2013) presented a detailed investigation of the variability of C IV and Si IV BALs on multi-year timescales in a large quasar sample assessing variation characteristics and the lifetimes of BAL troughs. This study found coordinated trough variability for BAL quasars showing multiple C IV troughs; they suggested that global changes in ionization level are the most straightforward mechanism for explaining such coordinated variability of multiple C IV troughs at different velocities. This mechanism would require at least some BAL troughs not to be highly saturated, as highly saturated troughs should not be responsive to the ex- pected changes in ionization level.

The available analytic calculations and numerical simulations of quasar disk winds predict the ionization level, kinematics, and column density of the outflowing gas along possible lines-of-sight to the relevant emission region (e.g., Murrav et al. 1995; Proga et al. 2000; Higginbottom et al.2013). These three quantities are expected generally to show correlated changes as the lineof-sight is varied. Thus, we expect correlated object-toobject changes of resulting observable phenomena such as the BAL transitions present, BAL-profile shapes, and BAL variability. In this paper, we aim to investigate systematically and quantify such correlated object-to-object changes for a large sample of BAL quasars with uniform high-quality measurements from the Sloan Digital Sky Survey (SDSS, York et al. 2000). Utilization of a large sample is important to overcome object-to-object scatter associated with, e.g., time-variable wind inhomogeneities.

To probe correlated object-to-object changes of ionization level, kinematics, and column density, we require a basic means of identifying lines-of-sight with different average ionization levels. We accomplish this using the strong BAL transitions of C IV, Si IV, and Al III. These three transitions span a significant range of ionization potential (with creation ionization potentials of $47.9,33.5$, and $18.8 \mathrm{eV}$, respectively, e.g., Hall et al. 2002), and their $\mathrm{BAL}$ regions can all be measured simultaneously in SDSS spectra of quasars with redshift $1.9<z<3.9$. Another practical advantage of using these three transitions is that their local continuum emission can be modeled more reliably than that for, e.g., Mg II. Lines-of-sight with C IV BALs but not Si IV or Al III BALs intercept only relatively highly ionized gas. Lines-of-sight with $\mathrm{C}$ IV and Si IV (but not Al III) BALs intercept at least some less ionized gas. Finally, lines-of-sight with BALs from all three ions intercept at least some even less ionized gas. In the numerical simulations of Proga et al. (2000), these three lines-of-sight lie at progressively larger inclinations relative to the rotational axis of the accretion disk.

In this study, utilizing multi-epoch observations from the SDSS (Section 2), we classify C IV BAL troughs into three groups considering the corresponding BAL regions of Si IV and Al III (Section 3). We present the observational results of our investigation in Section 4. In Section 5 , we present a summary of our results and a discussion of their implications for disk-wind models.

Throughout this study, timescales and EWs are given in the rest frame of the quasar unless stated otherwise. Negative signs for velocities indicate that a BAL trough is blueshifted with respect to the systemic velocity. We adapt a cosmology with $H_{0}=70 \mathrm{~km} \mathrm{~s}^{-1} \mathrm{Mpc}^{-1}, \Omega_{M}=0.3$, and $\Omega_{\Lambda}=0.7$ (e.g., Spergel et al. 2003).

\section{OBSERVATIONS, SAMPLE SELECTION, AND DATA PREPARATION}

We utilize spectroscopic observations from the Sloan Digital Sky Survey-I/II (hereafter SDSS, York et al. 2000) and the Baryon Oscillation Spectroscopic Survey of SDSS-III (hereafter BOSS, Eisenstein et al. 2011; Dawson et al. 2013). SDSS is a large multi-filter imaging and spectroscopic survey using a dedicated 2.5-m optical telescope (Gunn et al. 1998, 2006) at Apache Point Ob- 
servatory in New Mexico. During its first phase of operations, 2000-2005, the SDSS imaged more than $8000 \mathrm{deg}^{2}$ of the sky in five optical bandpasses, and it obtained spectra of galaxies and quasars. The SDSS spectral coverage was continuous from $3800 \AA$ to $9200 \AA$ at a resolution of 1800-2200 (e.g., York et al. 2000). In 2005 the survey entered a new phase, the SDSS-II, expanding its spectroscopic samples to over 800,000 galaxies and 100,000 quasars. The BOSS, part of the third phase of SDSS operations, is acquiring spectra for approximately 1.5 million luminous galaxies and 160,000 quasars (e.g., Anderson et al. 2012; Ross et al. 2012). The BOSS survey started operating in mid-2008 and is planned to continue observations until the end of June 2014. The BOSS quasar survey provides an outstanding opportunity for investigating intrinsic UV absorption in quasars, owing to its focus upon selection at $z>2.1$ which shifts the important C IV and Si IV transitions well into its spectral coverage (Smee et al. 2013). The BOSS spectral coverage is continuous from $3600 \AA$ to $10,000 \AA$ at a resolution of 1300-3000 (e.g., Dawson et al. 2013).

An ancillary BOSS project aims to investigate the dynamics of quasar winds over multi-year timescales utilizing second-epoch spectra for $2005 \mathrm{BAL}$ quasars originally identified in the SDSS-I/II spectroscopy by Gibson et al. (2009). These 2005 quasars were initially selected following $i<19.3,0.48<z<4.65, \mathrm{SN}_{1700}>6$, and $\mathrm{BI}_{0}>100 \mathrm{~km} \mathrm{~s}^{-1}$ criteria. Here $\mathrm{SN}_{1700}$ is the average signal-to-noise ratio in a $4 \AA$ resolution element within $1650-1750 \AA$, and $\mathrm{BI}_{0}$ is the modified "balnicity" index defined in Section 2 of Gibson et al. (2009). The details of the project and the target selection are described in Filiz Ak et al. (2012, 2013).

We select a sample of quasars for this study from these 2005 targets that were observed by SDSS between MJD 51,602 (2000 February 28) and 54,557 (2008 January 04) and by BOSS between MJD 55,176 (2009 December 11) and 56,455 (2013 June 12). Observation start dates correspond to completion of hardware commissioning for both SDSS and BOSS; post-commissioning observations have the most reliable spectral calibration. We applied basic spectral preparation procedures to these observed spectra following Section 3.1 of Filiz Ak et al. (2012) and select our "main sample" for this study considering the following criteria:

1. We select quasars with $1.9<z<3.9$ to ensure spectral coverage of both the Si IV 1394, $1403 \AA$ and $\mathrm{Al}$ III 1855, $1863 \AA$ absorption regions (out to $20,000 \mathrm{~km} \mathrm{~s}^{-1}$ ) where blueshifted absorption features are often found.

2. We select quasars that have a C IV balnicity index between -3000 and $-20,000 \mathrm{~km} \mathrm{~s}^{-1}, \mathrm{BI}_{3}^{20}$, greater than 0 for both the SDSS and BOSS spectra; implementing this requirement for both SDSS and BOSS spectra avoids biases that could arise from nonuniform SDSS vs. BOSS BAL-identification thresholds ${ }^{16}$ Thus the quasars with disappearing BAL troughs in Filiz Ak et al. (2012) are not included in

\footnotetext{
16 We have verified that only a small percentage of quasars $(\approx$ $10 \%$ ) have $\mathrm{BI}_{0}>100 \mathrm{~km} \mathrm{~s}^{-1}$ but $\mathrm{BI}_{3}^{20}=0$.
}

this study. We define $\mathrm{BI}_{3}^{20}$ using $a=3$ and $b=20$ for the generalized $\mathrm{BI}$ definition, $\mathrm{BI}_{a}^{b}$ :

$$
\mathrm{BI}_{a}^{b} \equiv \int_{-1000 \times a}^{-1000 \times b}\left(1-\frac{f(v)}{0.9}\right) C d v .
$$

Similar to the original BI definition (Weymann et al. 1991), in this equation $f(v)$ is the normalized flux density as a function of velocity, $v . C$ is a constant which is equal to 1.0 only when a trough is wider than $2000 \mathrm{~km} \mathrm{~s}^{-1}$; it is otherwise 0.0. Following the original BI definition, the minimum red-edge velocity limit is chosen to minimize confusion between CIV BALs and the C IV emission line. In this paper we use $\mathrm{BI}_{3}^{20}$, rather than $\mathrm{BI}_{3}^{30}$ used in Filiz Ak et al. (2013).

3 . We select only radio-quiet quasars by requiring the radio-loudness parameter, $R$, to be less than 10; we utilize $R$ parameters from Shen et al. (2011). Considering that radio-loud quasars are a minority part of the quasar population and may have different BAL properties than radio-quiet quasars (e.g., Becker et al. 1995, 2000; Brotherton et al. 1998), implementing this criterion avoids possible confusion associated with the presence of an additional radio-loud population.

Differing from the original BI definition of Wevmann et al. (1991), our adapted $\mathrm{BI}_{3}^{20}$ definition for this study (see Equation 11) limits the maximum blueedge velocity of a BAL-trough region at $20,000 \mathrm{~km} \mathrm{~s}^{-1}$, where it is $25,000 \mathrm{~km} \mathrm{~s}^{-1}$ in the original definition. Given that this study is focused on the characteristics of and differences between CIV BAL troughs that are accompanied by Si IV and/or Al III BALs in corresponding velocity ranges, we adjust the maximum blue-edge velocity limit of the C IV BAL-trough region considering the Si IV (1394, $1403 \AA)$ and Al III $(1855,1863 \AA)$ BALtrough regions. Both the Si IV and AlIII BAL-trough regions are occasionally contaminated by the emission lines of O I $1302 \AA$ (at $\approx-21,800 \mathrm{~km} \mathrm{~s}^{-1}$ from SiIV emission), Si II $1304 \AA$ (at $\approx-21,300 \mathrm{~km} \mathrm{~s}^{-1}$ from Si IV emission), $\mathrm{C}$ II $1334 \AA$ (at $\approx-14,500 \mathrm{~km} \mathrm{~s}^{-1}$ from Si IV emission), Ni II 1741 and $1751 \AA$ (at $\approx-20,200$ and $-18,400 \mathrm{~km} \mathrm{~s}^{-1}$ from Al III emission), and Fe II $1787 \AA$ (at $\approx-12,500 \mathrm{~km} \mathrm{~s}^{-1}$ from Al III emission). Although these emission lines are usually weak, a visual inspection showed that in some cases these features may bring an end to shallow BAL troughs. Moreover, low-velocity absorption from these other line species may lead to confusion in the detection of Si IV or Al III absorption. Thus, we consider a BAL-trough region between -3000 and $-20,000 \mathrm{~km} \mathrm{~s}^{-1}$ as a suitable balance between uncontaminated spectral regions and useful sample size. Both $\mathrm{C}$ II and Fe II have low ionization potentials $(24.4 \mathrm{eV}$ and $16.2 \mathrm{eV}$, respectively) and are rarely found in quasar spectra (e.g., Hall et al. 2002).

From the initial set of 2005 BAL quasars, the above criteria select 714 quasars that are observed by both the SDSS and BOSS (the main sample will be reduced to 
671 quasars below via further considerations). The median $\mathrm{SN}_{1700}$ is 10.7 for SDSS and 17.4 for BOSS observations. In order to compare multi-epoch observations of our main sample, for each spectrum, we follow the basic spectral preparation procedures given in Section 3.1 of Filiz Ak et al. (2012). These procedures include Galactic extinction correction using the $A_{V}$ values from Schlegel et al. (1998), transforming from the observed frame to the rest frame using the redshift values from Hewett \& Wild (2010), and removing pixels that are flagged by the SDSS and BOSS data-reduction pipelines (Bolton et al.|2012). As in Gibson et al. (2008, 2010) and Filiz Ak et al. (2012, 2013), we define relatively line-free (RLF) windows to reconstruct the underlying continuum. We fit the RLF windows of each spectrum with an intrinsically reddened power-law using a Small Magellanic Cloud type reddening model (e.g., Pei 1992). This fit is performed by running an iterative sigma-clipping algorithm where in each iteration we perform a non-linear least squares fit. We calculate the uncertainties on the continuum model using $\Delta \chi^{2}$ confidence-region estimation as described in Filiz Ak et al. (2012). We do not model the emission lines. We follow the procedures of Filiz Ak et al. (2012, 2013) for error propagation of continuum uncertainties into the subsequent measurements.

\section{IDENTIFICATION OF BAL TROUGHS AND MEASUREMENTS \\ 3.1. Identification of $B A L$ Troughs}

We identify C IV BAL troughs in the spectra of the 714 quasars that satisfy our quasar selection criteria (see Section 2 using the definition in Equation 1 and following the BAL-trough identification algorithm for multi-epoch observations defined in Section 3.2 of Filiz Ak et al. 2013). By construction, each quasar in our main sample has at least one SDSS and at least one BOSS observation. However, $\approx 25 \%$ of the main-sample quasars have multiple SDSS and/or BOSS observations. These repeat observations sample similar timescales (considering just the multi-year timescales of primary interest here) and could cause some of our objects to be given inordinate weight by allowing the repeat examination of BAL troughs. In order to avoid such multi-counting biases in our analyses, we use only two-epoch spectra for each quasar in our main sample. We select the one SDSS-I/II spectrum and the one BOSS spectrum that have the highest $\mathrm{SN}_{1700}$. Using these two-epoch observations, we classify C IV BAL troughs into three groups (denoted with subscripts) as explained below:

1. $\mathrm{CIV}_{00}$ indicates $\mathrm{CIV}$ BAL troughs with no detection of BAL or mini-BAL troughs at corresponding velocities in the Si IV and Al III absorption regions in both epochs.

2. $\mathrm{CIV}_{\mathrm{So}}$ indicates $\mathrm{C}$ IV BAL troughs accompanied by a Si IV BAL trough in either epoch but with no detection of a BAL or mini-BAL trough at corresponding velocities in the $\mathrm{Al}$ III BAL region 17

17 We define a mini-BAL using Equation 1 but adapting the constant, $C$, equal to one for absorption lines with $500<\Delta v<$ $2000 \mathrm{~km} \mathrm{~s}^{-1}$ and zero otherwise (e.g., [Filiz Ak et al.]2013, and references therein).
3. $\mathrm{CIV}_{\mathrm{SA}}$ indicates $\mathrm{C}$ IV BAL troughs accompanied by a Si IV BAL and also an AlIII BAL detected at corresponding velocities in either epoch.

We did not find any examples of C IV BAL troughs accompanied by an Al III BAL but no Si IV BAL (i.e., $\mathrm{CIV}_{0 \mathrm{~A}}$ ); lower ionization absorption (in this case, Al III) troughs are always accompanied by higher ionization (Si IV and C IV) troughs at the same velocities. As discussed in Section 1, these three C IV groups serve as a basic means for identifying lines-of-sight with different average ionization levels.

We identify 43 quasars showing multiple CIV BAL troughs that are classed in different $\mathrm{C}$ IV groups (38 with $\mathrm{CIV}_{00}$ and $\mathrm{C}_{\mathrm{IV}} \mathrm{So}$ troughs, and 5 with $\mathrm{C} \mathrm{IV}_{\mathrm{S} 0}$ and $\mathrm{C} \mathrm{IV}_{\mathrm{SA}}$ troughs). While the existence of such objects is entirely expected within accretion-disk wind models, practically they introduce complexity in connecting a given BAL trough to a given line-of-sight; e.g., $\mathrm{C}_{\mathrm{IV}_{00}}$ troughs in quasars that also show $\mathrm{C} \mathrm{IV}_{\mathrm{S} 0}$ troughs likely sample a different part of the outflow from $\mathrm{CIV}_{00}$ troughs in quasars with no other BALs (see Section [5] for further discussion). To reduce complexity and avoid potentially mixing troughs in the same C IV group that sample different parts of the outflow, we exclude from our main sample these 43 quasars. We have performed all analyses below also including such quasars, and the results do not change materially. After excluding these 43 quasars, there are 671 quasars in our main sample (see Table1). We present our $\mathrm{BI}_{3}^{20}$ measurements and uncertainties on $\mathrm{BI}_{3}^{20}, \sigma_{\mathrm{BI}_{3}^{20}}$, both for SDSS and BOSS observations of BAL quasars in our main sample in Table 1] $\sigma_{\mathrm{BI}_{3}^{20}}$ is propagated from uncertainties on the estimated continuum and errors on the trough measurements. We detect a total of $852 \mathrm{C}$ IV BAL troughs in the spectra of these 671 main-sample quasars as our main-sample $\mathrm{C}$ IV troughs.

We have identified $113 \mathrm{CIV}_{00}, 246 \mathrm{CIV}_{\mathrm{S} 0}$, and 95 $\mathrm{CIV}_{\mathrm{SA}} \mathrm{BAL}$ troughs in our main sample. We present our measurements for $\mathrm{CIV}_{00}$ troughs in Table 2. $\mathrm{CIV}_{\mathrm{S} 0}$ and corresponding Si IV BAL troughs in Table 3, and $\mathrm{CIV}_{\mathrm{SA}}$ and corresponding Si IV and Al III BAL troughs in Table 4. In addition to these groups we have detected 271 C IV BAL troughs accompanied by a Si IV mini-BAL $\left(\mathrm{C}_{\mathrm{IV}} \mathrm{S} 0\right.$, where the lower case subscript "s" denotes the mini-BAL nature), $32 \mathrm{C}$ IV BAL troughs accompanied by a Si IV mini-BAL and also an Al III mini-BAL ( $\left.\mathrm{C} \mathrm{IV}_{\mathrm{sa}}\right)$, and $94 \mathrm{C}$ IV BAL troughs accompanied by a Si IV BAL and an Al III mini-BAL ( $\left.\mathrm{CIV}_{\mathrm{Sa}}\right)$. We present our measurements for $\mathrm{CIV}_{\mathrm{s} 0}$ troughs in Table [5, $\mathrm{CIV}_{\mathrm{sa}}$ troughs in Table 6, and $\mathrm{C}_{\mathrm{IV}_{\mathrm{Sa}}}$ troughs in Table 7 . We do not include these troughs in any of the groups defined above to consider only strong intrinsic absorption of the given transition; this approach should give the strongest distinction between groups.

We have cross-matched our 671 main-sample quasars with the SDSS DR7 quasar properties catalog of Shen et al. (2011) to obtain absolute $i$-band magnitudes, $M_{i}$ (see Table 11). We found that the majority of our main-sample quasars lie within $2.5 \mathrm{mag}$ in $M_{i}$, corresponding to a factor of $\approx 10$ range in $i$-band luminosity. Given this relatively narrow range, we do not have any immediate reason to be concerned about luminosity effects. Moreover, using two-sample Anderson-Darling (AD) tests (see Press et al. 2007), we found that the $M_{i}$ 
distributions for the $\mathrm{CIV}_{00}, \mathrm{CIV}_{\mathrm{S} 0}$, and $\mathrm{CIV}_{\mathrm{SA}}$ groups are consistent, indicating that luminosity effects should not be causing confusion in group intercomparisons.

Figures 1, 2, and 3 show two-epoch observations for representative examples of $\mathrm{CIV}_{00}, \mathrm{CIV}_{\mathrm{S} 0}$, and $\mathrm{CIV}_{\mathrm{SA}}$ BAL troughs, respectively. We smoothed each spectrum for display purposes using a Savitzky-Golay algorithm (see Press et al. 2007) that performs a local linear regression for three consecutive points (see Filiz Ak et al. 2012). In these figures, we indicate CIV BAL troughs and their corresponding velocity ranges in the Si IV and $\mathrm{Al}$ III absorption regions. The SDSS identification, redshift, and timescale between the two epochs are given in each panel.

In this study, we generally focus on the C IV, Si IV, and $\mathrm{Al}$ III BAL regions. We do not analyze in detail the BAL regions of the other transitions listed in Section 1 for several reasons: (1) Due to the limited wavelength coverage of the observed spectra (3800-9200 $\AA$ for SDSS, and $3600-10000 \AA$ for BOSS), only a small number of quasars have suitable wavelength coverage to investigate all of these transitions. (2) The strong $\operatorname{Ly} \alpha$ and $\mathrm{N} v$ lines blend with each other and their blueshifted absorption lines blend with Ly $\alpha$ forest, where numerous absorption features from intervening gas are found. (3) A proper investigation of Mg II BALs would require modeling and subtraction of broad Fe II emission in addition to underlying continuum estimation. Moreover, only a small fraction of our targeted quasars have the wavelength coverage to investigate simultaneously CIV, Si IV, and Mg II BAL troughs.

\subsection{Measurements of BAL Troughs}

We measure the rest-frame timescales, $\Delta t$, for our main sample; $\Delta t$ is between 0.85 and $4.13 \mathrm{yr}$ with a mean of 2.55 yr. Given the established connection between BALvariation strength and timescale (e.g., Gibson et al.|2010; Filiz Ak et al. 2013), we compared the $\Delta t$ distributions for $\mathrm{CIV}_{00}, \mathrm{C}_{\mathrm{IV}} \mathrm{SO}$, and $\mathrm{C}_{\mathrm{IV}} \mathrm{SA}$ troughs. In Figure 4, we show the $\Delta t$ distributions for all $\mathrm{C}$ IV troughs in our main sample and for $\mathrm{C}_{\mathrm{IV}} 00, \mathrm{C}_{\mathrm{IV}} \mathrm{S}$, and $\mathrm{C}_{\mathrm{IV}} \mathrm{SA}$ troughs. Given their definition, the sum of the total number of $\mathrm{CIV}_{00}$, $\mathrm{C}_{\mathrm{IV}} \mathrm{S}$, and $\mathrm{C}_{\mathrm{IV}} \mathrm{SA}$ troughs is not equal to the total number of C IV troughs in our sample. We compared these $\Delta t$ distributions using two-sample $\mathrm{AD}$ tests. The test results show no significant differences between the distributions.

Considering that BAL troughs are sometimes isolated and occasionally appear in complexes in which single troughs may split or adjacent troughs may merge over time, we define minimum and maximum velocities for each BAL trough following the BAL-trough identification algorithm for multi-epoch observations described in Section 3.2 of Filiz Ak et al. (2013). We set $v_{\min }$ to be the minimum red-edge velocity and $v_{\max }$ to be the maximum blue-edge velocity of the associated absorption complex in all available epochs. For BAL troughs that reach beyond our adopted velocity limits (see Equation (1), we simply truncate the BAL trough $v_{\max }$ to be $-20,000 \mathrm{~km} \mathrm{~s}^{-1}$ and $v_{\min }$ to be $-3000 \mathrm{~km} \mathrm{~s}^{-1}$.

We measure the rest-frame EW of each BAL trough in each epoch. To calculate uncertainties on EWs, we propagate observational errors for each contributing pixel and continuum-estimation errors (see Filiz Ak et al. 2012,
2013) using Equations 1 and 2 of Kaspi et al. (2002). We calculate $\mathrm{EW}$ variations, $\Delta \mathrm{EW}$, fractional $\mathrm{EW}$ variations, $\Delta \mathrm{EW} /\langle\mathrm{EW}\rangle$, and uncertainties on these quantities, $\sigma_{\Delta \mathrm{EW}}$ and $\sigma_{\Delta \mathrm{EW} /\langle\mathrm{EW}\rangle}$, following Equations 3 and 4 of Filiz Ak et al. (2013), respectively. In this study, positive values of $\Delta \mathrm{EW}$ and $\Delta \mathrm{EW} /\langle\mathrm{EW}\rangle$ indicate strengthening troughs and negative values indicate weakening troughs. In addition, we measure the average depth, $d_{\mathrm{BAL}}$, for each trough by calculating the mean distance of each contributing data point from the normalized continuum level. We calculate a BAL-trough velocity width, $\Delta v$, and a weighted centroid velocity, $v_{\text {cent }}$, which is the mean velocity where each data point is weighted with its distance from the normalized continuum level. We adapt redshift values from Hewett \& Wild (2010) for all velocity calculations.

\subsection{Comparisons with $\mathrm{Mg} \mathrm{II}, \mathrm{Fe} \mathrm{II}$, and $\mathrm{P}_{\mathrm{V}}$}

We have compared our adopted classification of C IV BAL troughs with the standard subtypes of HiBALs, LoBALs, and FeLoBALs (see Section [1). All $113 \mathrm{CIV}_{00}$ and $246 \mathrm{C}$ IV $_{\mathrm{So}}$ troughs fit into the definition of HiBALs where only BAL troughs of high-ionization transitions are present in the spectra. Standard classes of LoBALs and FeLoBALs are identified with the presence of $\mathrm{Mg}$ II and Fe II absorption, respectively. We visually investigated the $\mathrm{Mg}$ II $\lambda \lambda 2797,2804 \AA$ and Fe II $\lambda \lambda 2400$, $2600 \AA$ absorption-line regions for $95 \mathrm{C} \mathrm{IV}_{\mathrm{SA}}$ troughs to examine the correspondence to the standard LoBAL and FeLoBAL definitions. The spectra of 34 quasars with $\mathrm{C}_{\text {IV }}$ SA troughs do not have coverage of the Mg II region, and these are not utilized in our correspondence checking. We find that $\approx 74 \%$ ( 45 out of 61 ) of quasars with $\mathrm{C}_{\text {IV }}$ SA troughs exhibit Mg II BAL/mini-BAL troughs at corresponding velocities; four of them also exhibit Fe II $\mathrm{BAL} /$ mini-BAL troughs. There are 16 quasars with $\mathrm{C}_{\text {IV }}$ SA troughs that have no $\mathrm{Mg}$ II absorption. Due to the differences between their ionization potentials $(28.4 \mathrm{eV}$ for $\mathrm{Al}$ III and $15.0 \mathrm{eV}$ for $\mathrm{Mg} \mathrm{II}), \mathrm{Al}$ III absorption is expected to be slightly more common than Mg II absorption given that higher ionization troughs are found more frequently than lower ionization troughs in BAL quasars (e.g., Hall et al. 2002).

Previous investigations of $\mathrm{P} v \lambda \lambda 1118,1128 \AA$ absorption lines corresponding in velocity with $\mathrm{CIV}$ and $\mathrm{Si}$ IV $\mathrm{BAL}$ troughs have shown that $\mathrm{P} \mathrm{V}$ absorption lines are an important indicator of line saturation (e.g., Hamann 1998; Arav et al. 2001); note that the ionization potentials of $\mathrm{P} \mathrm{V}(65.0 \mathrm{eV})$ and $\mathrm{C} \mathrm{IV}(64.5 \mathrm{eV})$ are very similar. We thus visually investigate the $\mathrm{P} v$ BAL regions that align with our $\mathrm{CIV}_{00}, \mathrm{C}_{\mathrm{IV}} \mathrm{S}$, and $\mathrm{CIV}_{\mathrm{SA}}$ troughs. The spectral coverage is sufficient to investigate the $\mathrm{P} v \mathrm{BAL}$ region for $40 \mathrm{CIV}_{00}$ troughs, $113 \mathrm{C}_{\mathrm{IV}} \mathrm{So}$ troughs, and $47 \mathrm{CIV}_{\mathrm{SA}}$ troughs. Our visual inspection reveals that spectra of a large fraction $(\approx 88 \%)$ of $\mathrm{C} \mathrm{IV}_{\mathrm{SA}}$ troughs exhibit visually detectable $\mathrm{P} \mathrm{V}$ absorption features; a majority $(\approx 70 \%)$ of $\mathrm{CIV}_{\mathrm{SA}}$ troughs are accompanied by moderate-to-strong $\mathrm{P} v$ absorption. $\mathrm{P} v$ absorption that aligns with $\mathrm{CIV}_{\mathrm{S} 0}$ troughs is generally weaker than that aligning with $\mathrm{C}_{\mathrm{IV}} \mathrm{SA}$ troughs. Approximately half of the $\mathrm{C}_{\text {IV }}$ So troughs are accompanied by detectable $\mathrm{PV}$ absorption; however, only $\approx 10 \%$ of those $\mathrm{CIV}_{\mathrm{S} 0}$ troughs align with moderate-to-strong $\mathrm{P}$ V absorption. Only a 

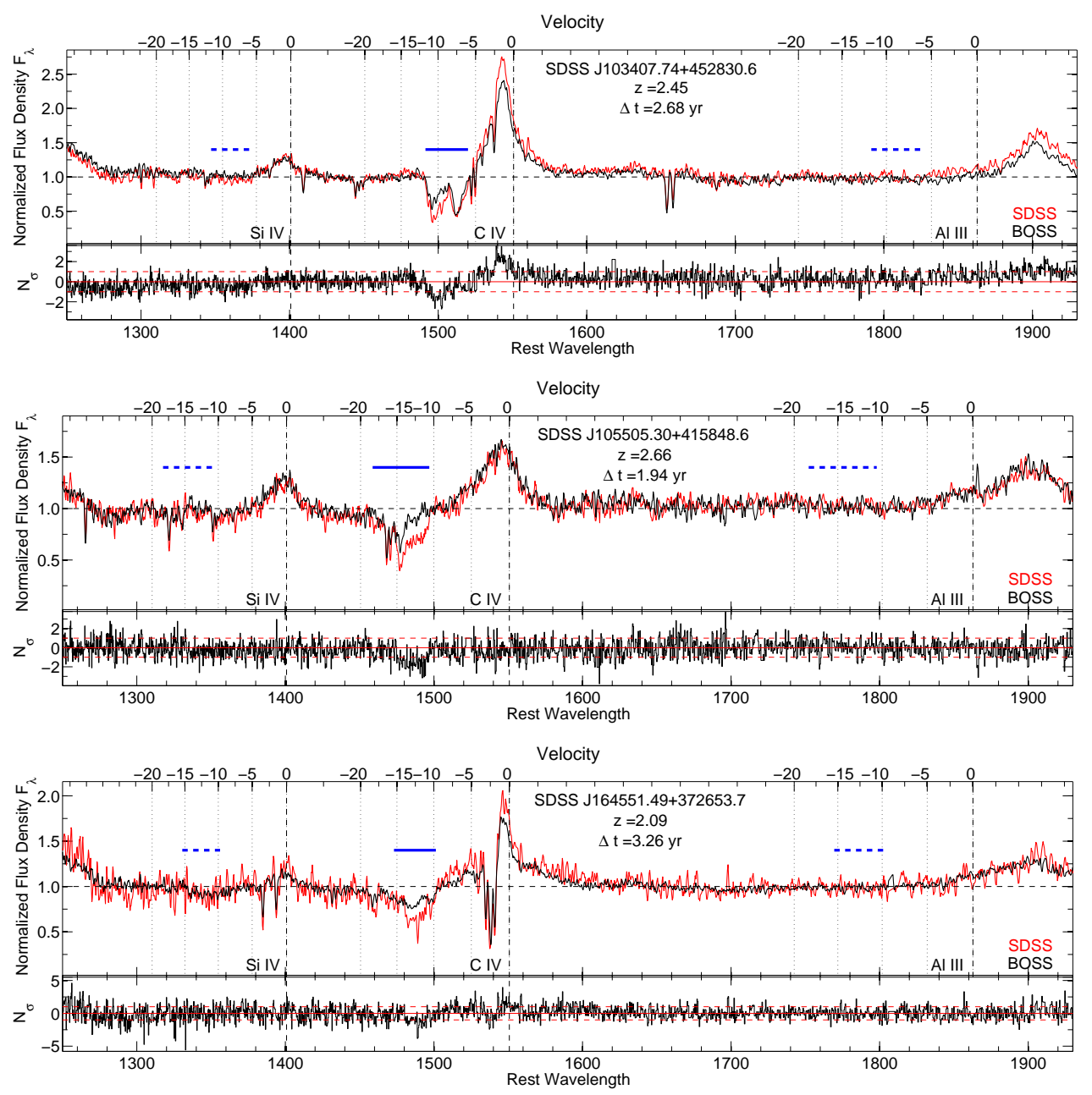

Figure 1. Two-epoch spectra of quasars with $\mathrm{C}_{\mathrm{IV}} \mathrm{D}_{00} \mathrm{BAL}$ troughs (i.e., C IV BAL troughs with no detection of BAL or mini-BAL troughs at corresponding velocities in the SiIV and Al III absorption regions) from SDSS (red) and BOSS (black). The $x$-axes show both the rest-frame wavelength (bottom, in $\AA$ ) and the blueshift velocity from the Si IV, C IV, and Al III emission lines (top, in $10^{3} \mathrm{~km} \mathrm{~s}^{-1}$ ). The $y$-axes show flux densities normalized by the fitted continuum model $\left(F_{\lambda}\right)$. The horizontal solid-blue bars designate CIV BAL troughs, and the dashed blue bars indicate corresponding velocities in the Si IV and Al III BAL regions. The lower section of each panel shows deviations between SDSS and BOSS observations for each $\approx 4 \AA$ pixel in units of $\sigma, N_{\sigma}$, and the dashed-red lines show $\pm 1 \sigma$ levels.

small fraction $(\approx 12 \%)$ of $\mathrm{CIV}_{00}$ troughs are accompanied by detectable $\mathrm{P}_{\mathrm{V}}$ absorption, and the strength of this $\mathrm{P}$ V absorption is generally weak. These results show that quasars possessing detectable $\mathrm{P} v$ absorption in their spectra are also more likely to present lower-ionization transitions; therefore their C IV troughs are likely to be $\mathrm{CIV}_{\mathrm{SA}}$ or to a smaller extent $\mathrm{C}_{\mathrm{IV}_{\mathrm{S} 0}}$ troughs.

\section{RESULTS}

In this section, we present the observational results of our investigation. Utilizing the two-epoch observations for $113 \mathrm{CIV}_{00}, 246 \mathrm{C}_{\mathrm{IV}}$, and $95 \mathrm{C}_{\mathrm{IV}} \mathrm{SA} \mathrm{BAL}$ troughs, we investigate the CIV, SiIV, and Al III BAL profiles (Section 4.1), the C IV trough strengths (Section 4.2), the C IV trough velocities (Section 4.3), the C IV trough variation profiles (Section 4.4), the C IV trough $\mathrm{EW}$ variation characteristics (Section 4.5), and the C IV, Si IV, and Al III trough EW variation correlations (Section 4.6).

\subsection{BAL-Trough Profiles}

It is well known that BAL troughs from different transitions show different profiles; for instance,
Al III BAL troughs tend to be narrower and align with lower velocity portions of corresponding C IV BAL troughs (e.g., Weymann et al. 1991; Voit et al. 1993; Trump et al. 2006). Moreover, previous studies (e.g., Weymann et al. 1991; Reichard et al. 2003; Allen et al. 2011) have demonstrated that C IV BAL troughs tend to be stronger in quasars exhibiting $\mathrm{Al}$ III and/or Mg II BAL troughs (i.e., LoBAL quasars). To compare the typical properties of $\mathrm{CIV}_{00}, \mathrm{CIV}_{\mathrm{S} 0}$, and $\mathrm{CIV}_{\mathrm{SA}} \mathrm{BAL}$ troughs, we calculate composite mean profile shapes for each C IV group. Figure 5] shows the mean profile shapes for 113 $\mathrm{CIV}_{00}, 246 \mathrm{CIV}_{\mathrm{S} 0}$, and $95 \mathrm{CIV}_{\mathrm{SA}} \mathrm{BAL}$ troughs as a function of outflow velocity relative to $v_{\text {min }}$. We calculate the mean profiles by defining an outflow velocity, $v_{\mathrm{t}}=v-v_{\min }$, which is set to 0 at its $v_{\min }$ and runs from 0 to $\Delta v$ (where $\Delta v$ is the trough velocity width). We fix the normalized flux density, $F_{\lambda}$, to 1 at velocities higher than $v_{\max }$ for each C IV BAL trough. Given the BALtrough definition, $F_{\lambda}$ is less than 0.9 for $0<v_{\mathrm{t}}<\Delta v$. We also present mean profile shapes of Si IV and Al III BAL troughs in overlapping velocity ranges for comparison.

A comparison of the resulting composite profiles in Fig- 

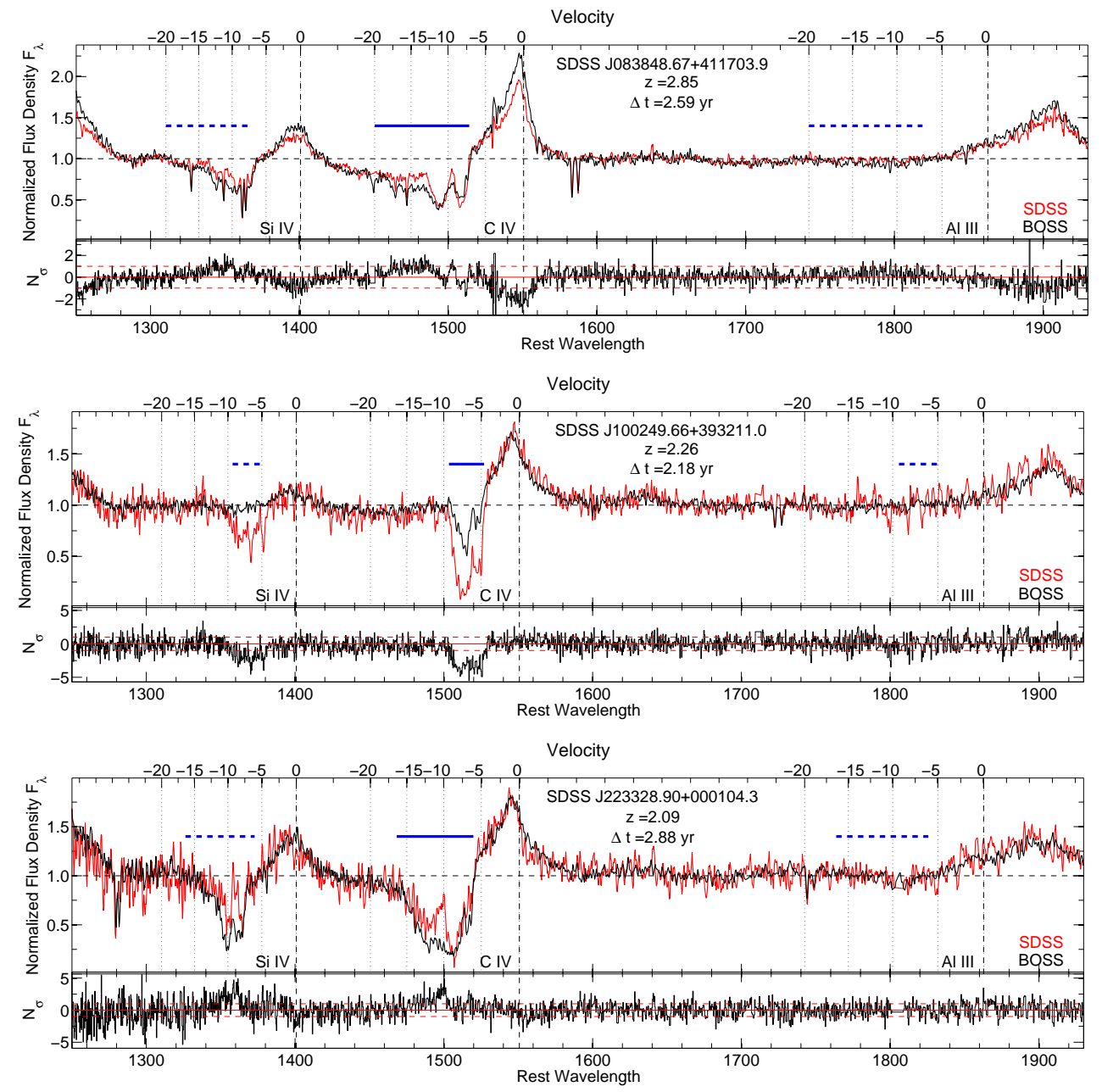

Figure 2. Same as Figure 1 but for $\mathrm{CIV}_{\mathrm{SO}} \mathrm{BAL}$ troughs (i.e., C IV BAL troughs accompanied by a Si IV BAL trough but with no detection of a BAL or mini-BAL trough at corresponding velocities in the Al III BAL region).

ure [5]indicates that $\mathrm{C}_{\mathrm{IV}} 00$ troughs tend to be shallower and narrower than both $\mathrm{CIV}_{\mathrm{S} 0}$ and $\mathrm{CIV}_{\mathrm{SA}}$ troughs. The $\mathrm{CIV}_{\mathrm{S} 0}$ and $\mathrm{CIV}_{\mathrm{SA}}$ trough depths change in a characteristic manner as a function of velocity within the trough: troughs are generally deeper at lower velocities. Consistent with previous studies, the composite profile shapes show that CIV BALs are usually deepest when there is an Al III BAL at corresponding velocities. Similarly, a Si IV BAL-trough profile change is apparent between the $\mathrm{C} I V_{\text {So }}$ and $\mathrm{CIV}_{\mathrm{SA}}$ samples. We also find that $\mathrm{Al}$ III BAL troughs tend to be narrower and be found at lower velocities than corresponding $\mathrm{C}_{\mathrm{IV}_{\mathrm{SA}}} \mathrm{BAL}$ troughs.

We find qualitatively consistent results when computing median composites instead of mean composites. This indicates that outliers are not strongly affecting our composites.

\subsection{BAL-Trough Strengths}

For a more quantitative comparison between the $\mathrm{CIV}_{00}, \mathrm{C}_{\mathrm{IV}} \mathrm{S}$, and $\mathrm{C} \mathrm{IV}_{\mathrm{SA}}$ groups, we assess differences between measured CIV BAL-trough properties in this subsection and the next. Figure 6 shows the distributions of average $\mathrm{EW}$ from two-epoch observations, $\langle\mathrm{EW}\rangle$, for $\mathrm{C}_{\mathrm{IV}_{00}}, \mathrm{C}_{\mathrm{IV}} \mathrm{S} 0$, and CIV $\mathrm{CA}$ BAL troughs. Considering that the total number of BAL troughs increases with decreasing $\langle\mathrm{EW}\rangle$, the fraction of BAL troughs with given
$\langle\mathrm{EW}\rangle$ is also displayed in Figure 6. We calculate the fractions as the ratio of the number of $\mathrm{CIV}_{00}, \mathrm{CIV}_{\mathrm{SO}}$, and $\mathrm{CIV}_{\mathrm{SA}} \mathrm{BAL}$ troughs to all 852 main-sample C IV troughs; they therefore need not sum to unity in a given bin.

The mean $\langle\mathrm{EW}\rangle$ is $15.46 \pm 0.42 \AA$ for all $852 \mathrm{C}$ IV BAL troughs in our main sample, whereas it is $4.76 \pm 0.25 \AA$ for $\mathrm{CIV}_{00}, 19.29 \pm 0.62 \AA$ for $\mathrm{CIV}_{\mathrm{S} 0}$, and $32.38 \pm 1.35 \AA$ for $\mathrm{C}_{\mathrm{IV}} \mathrm{SA}$ troughs as given in Table 8 . Uncertainties on the mean are calculated using the standard $\sigma / \sqrt{N}$ formula. The median $\langle\mathrm{EW}\rangle$ is $11.51 \AA$ for all $\mathrm{C}$ IV, $4.44 \AA$ for $\mathrm{CIV}_{00}, 17.58 \AA$ for $\mathrm{CIV}_{\mathrm{S} 0}$, and $31.14 \AA$ for $\mathrm{CIV}_{\mathrm{SA}}$ BAL troughs. These results confirm and quantify the increase of C IV BAL-trough strength with the existence of absorption lines from lower ionization-level transitions.

In order to determine the contributions of the depth and width components of BAL EWs, we assess average depth from two-epoch observations, $\left\langle d_{\mathrm{BAL}}\right\rangle$, and velocity width, $\Delta v$, distributions for $\mathrm{C}_{\mathrm{IV}_{00}}, \mathrm{C}_{\mathrm{IV}} \mathrm{S}_{\mathrm{S}}$, and $\mathrm{C}_{\mathrm{IV}} \mathrm{SA}$ troughs (see Figure 7). Table 8 presents the mean $\left\langle d_{\mathrm{BAL}}\right\rangle$ and $\Delta v$ values for all three C IV BAL-trough groups. We compare these distributions using an $\mathrm{AD}$ test and find that both the $\left\langle d_{\mathrm{BAL}}\right\rangle$ and $\Delta v$ distributions for $\mathrm{C}_{\mathrm{IV}_{00}}$, $\mathrm{CIV}_{\mathrm{S} 0}$, and $\mathrm{CIV}_{\mathrm{SA}}$ BAL troughs are significantly different (at a confidence level of $>99.9 \%$ ) from each other. 

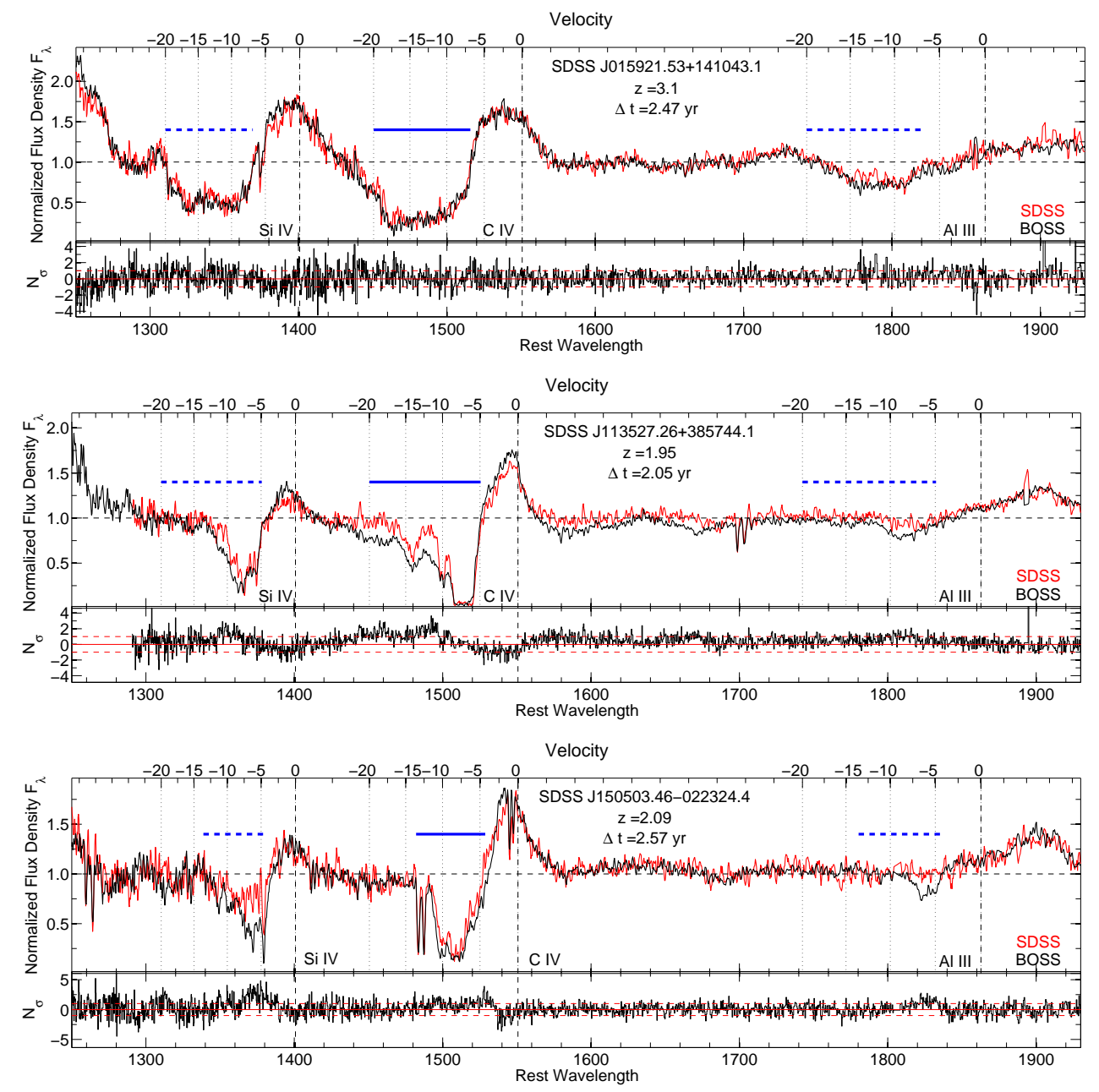

Figure 3. Same as Figure 1 but for $\mathrm{CIV}_{\mathrm{SA}}$ BAL troughs (i.e., C IV BAL troughs accompanied by a Si IV BAL trough and also an Al III BAL trough).

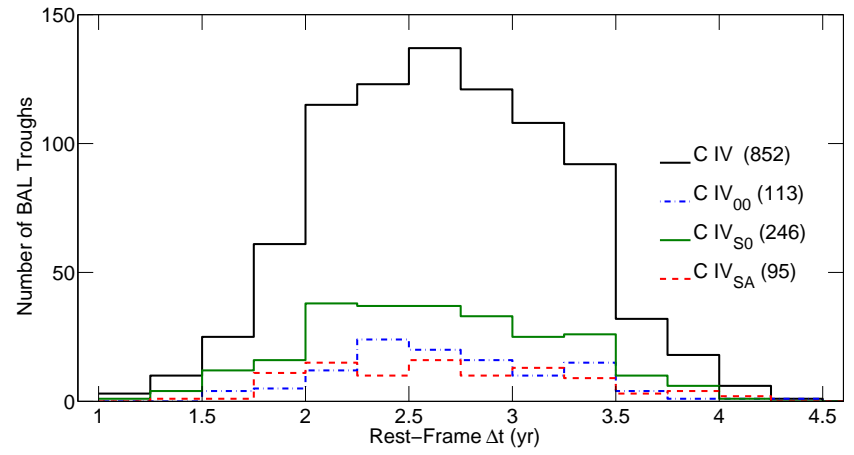

Figure 4. The rest-frame $\Delta t$ distributions for all $\mathrm{C}$ IV troughs in our main sample (solid black) and $\mathrm{CIV}_{00}$ (dot-dashed blue), $\mathrm{C}_{\text {IVSo }}$ (dashed green), and CIVSA (solid red) BAL troughs. The total number of BAL troughs in each sample is given in parentheses. After renormalization for the number of each type of trough, there are no statistically significant differences between the distributions. Given their definitions, the sum of the total numbers of $\mathrm{CIV}_{00}, \mathrm{CIV}_{\mathrm{S} 0}$, and $\mathrm{CIV}_{\mathrm{SA}}$ troughs are not necessarily equal to total number of $\mathrm{C}$ IV troughs in our main sample. The AndersonDarling test results indicate no significant $\Delta t$ differences between the samples.

Our findings indicate that $\mathrm{CIV}_{\mathrm{SA}} \mathrm{BAL}$ troughs tend to be the deepest and widest BAL troughs, while $\mathrm{CIV}_{00}$ troughs tend to be the shallowest and narrowest. The ranges of the $\left\langle d_{\mathrm{BAL}}\right\rangle$ and $\Delta v$ values for $\mathrm{CIV}_{00}, \mathrm{CIV}_{\mathrm{S} 0}$, and $\mathrm{CIV}_{\mathrm{SA}} \mathrm{BAL}$ troughs demonstrate that the contributions of the depth and the width to the differences between BAL-trough EWs are comparable; the $\left\langle d_{\mathrm{BAL}}\right\rangle$ values change by a factor of $\approx 2.3$ and the $\Delta v$ values change by a factor of $\approx 3$ between the $\mathrm{CIV}_{00}$ and $\mathrm{CIV}_{\mathrm{SA}}$ samples.

Note that for BAL troughs extending beyond our adopted BAL-trough definition velocity limits, the $v_{\min }$ and $v_{\max }$ values are truncated at -3000 and $-20,000 \mathrm{~km} \mathrm{~s}^{-1}$, respectively. Due to this truncation process, the measured $\Delta v$ values are only lower limits for such BAL troughs. However, the results discussed in Section 4.3 indicate that such truncation does not have a strong effect on our main conclusions.

As can be seen in Figures [5, the strength of Si IV $\mathrm{BAL}$ troughs is also larger when $\mathrm{Al}$ III BAL troughs are present. The mean $\langle\mathrm{EW}\rangle$ is $7.35 \pm 0.49 \AA$ for $\mathrm{Si}$ IV BAL troughs in the $\mathrm{CIV}_{\mathrm{S} 0}$ sample and $20.84 \pm 1.02 \AA$ for Si IV BAL troughs in the $\mathrm{CIV}_{\mathrm{SA}}$ sample. The mean $\langle\mathrm{EW}\rangle$ values change by a factor of $\approx 2.8$, which is even stronger than the corresponding change for $\mathrm{C}$ IV of $\approx 1.7$.

\subsection{BAL-Trough Velocities}

To assess differences in BAL-trough velocities, we investigate minimum velocity, $v_{\min }$, maximum velocity, 
The Dependence of Civ BAL Properties on Accompanying Si iv and Al ini BALs

Table 1

Main-Sample BAL Quasars

\begin{tabular}{|c|c|c|c|c|c|c|c|}
\hline Quasar ID & $\begin{array}{l}\text { Quasar Name } \\
\text { SDSS }\end{array}$ & $\begin{array}{l}\text { RA } \\
\text { J2000 }\end{array}$ & $\begin{array}{l}\text { Dec } \\
\text { J2000 }\end{array}$ & $z$ & $\sigma_{z}$ & $\begin{array}{l}M_{\mathrm{i}}(z=2) \\
\quad(\mathrm{mag})\end{array}$ & Plate[1] \\
\hline Q1 & J000119.64+154828.8 & 0.33184 & 15.80800 & 1.921029 & 0.000494 & -26.73 & 750 \\
\hline Q2 & $\mathrm{J} 001025.90+005447.6$ & 2.60796 & 0.91324 & 2.859854 & 0.000323 & -27.81 & 389 \\
\hline Q3 & $\mathrm{J} 001502.26+001212.4$ & 3.75943 & 0.20346 & 2.852539 & 0.000552 & -27.68 & 389 \\
\hline Q4 & $\mathrm{J} 003135.57+003421.2$ & 7.89823 & 0.57257 & 2.236426 & 0.000255 & -27.65 & 689 \\
\hline Q5 & $\mathrm{J} 003312.25+155442.4$ & 8.30105 & 15.91178 & 1.955444 & 0.000617 & -26.74 & 417 \\
\hline Q6 & J003517.95+004333.7 & 8.82481 & 0.72604 & 2.916893 & 0.000545 & -27.61 & 1086 \\
\hline Q7 & J003551.98+005726.4 & 8.96660 & 0.95734 & 1.905903 & 0.000576 & -26.75 & 392 \\
\hline Q8 & J003832.26+152515.5 & 9.63446 & 15.42100 & 2.448965 & 0.000500 & -27.46 & 418 \\
\hline Q9 & $\mathrm{J} 004732.73+002111.3$ & 11.88639 & 0.35315 & 2.873223 & 0.000340 & -27.99 & 691 \\
\hline Q10 & J005215.64+003120.5 & 13.06520 & 0.52236 & 2.792256 & 0.000321 & -27.48 & 084 \\
\hline
\end{tabular}

\begin{tabular}{|c|c|c|c|c|c|c|c|c|c|c|c|}
\hline $\begin{array}{c}\text { MJD[1] } \\
\text { (days) }\end{array}$ & Fiber[1] & $\mathrm{SN}_{1700}[1]$ & Plate[2] & $\begin{array}{c}\text { MJD[2] } \\
\text { (days) }\end{array}$ & Fiber[2] & $\mathrm{SN}_{1700}[2]$ & $\begin{array}{r}\mathrm{BI}_{3}^{20}[1] \\
\left(\mathrm{km} \mathrm{s}^{-1}\right)\end{array}$ & $\begin{array}{r}\sigma_{\mathrm{BI}_{3}^{20}}[1] \\
\left(\mathrm{kms}^{-1}\right)\end{array}$ & $\begin{array}{r}\mathrm{BI}_{3}^{20}[2] \\
\left(\mathrm{kms}^{-1}\right)\end{array}$ & $\begin{array}{r}\sigma_{\mathrm{BI}_{3}^{20}}[2] \\
\left(\mathrm{kms}^{-1}\right)\end{array}$ & $\begin{array}{c}\Delta t \\
\text { (years) }\end{array}$ \\
\hline 52235 & 566 & 8.201 & 6172 & 56269 & 318 & 18.296 & 6378 & 142 & 7291 & 92 & 3.781 \\
\hline 51795 & 332 & 6.379 & 4218 & 55479 & 592 & 20.610 & 3974 & 212 & 4893 & 77 & 2.613 \\
\hline 51795 & 465 & 10.623 & 4218 & 55479 & 818 & 21.430 & 2273 & 107 & 2042 & 28 & 2.618 \\
\hline 52262 & 502 & 15.060 & 3587 & 55182 & 570 & 24.501 & 5305 & 39 & 5855 & 23 & 2.470 \\
\hline 51821 & 576 & 8.304 & 6192 & 56269 & 184 & 15.174 & 11666 & 168 & 11571 & 91 & 4.121 \\
\hline 52525 & 481 & 12.271 & 3588 & 55184 & 512 & 14.787 & 1811 & 70 & 1406 & 44 & 1.859 \\
\hline 51793 & 449 & 8.931 & 3588 & 55184 & 552 & 24.975 & 2724 & 66 & 2194 & 27 & 3.195 \\
\hline 51817 & 483 & 6.585 & 6197 & 56191 & 760 & 12.239 & 7509 & 174 & 9076 & 114 & 3.472 \\
\hline 52199 & 559 & 19.051 & 4223 & 55451 & 724 & 26.027 & 1165 & 39 & 1045 & 23 & 2.299 \\
\hline 52591 & 516 & 9.739 & 4223 & 55451 & 968 & 11.653 & 4709 & 118 & 7004 & 146 & 2.065 \\
\hline
\end{tabular}

Notes. Throughout this table [1] indicates the first-epoch spectra and [2] indicates the second-epoch spectra.

(This table is available in its entirety in a machine-readable form in the online journal. A portion is shown here for guidance regarding its form and content.)

$v_{\max }$, and average centroid velocity from two-epoch observations, $\left\langle v_{\text {cent }}\right\rangle=1 / 2\left(v_{\text {cent }_{1}}+v_{\text {cent }_{2}}\right)$, for $\mathrm{C}_{\mathrm{IV}_{00}}$, $\mathrm{CIV}_{\mathrm{S} 0}$, and $\mathrm{CIV}_{\mathrm{SA}} \mathrm{BAL}$ troughs. We present these distributions in Figure 8 .

Given our adopted BAL-trough definition, $v_{\min }$ and $v_{\max }$ values are truncated at -3000 and $-20,000 \mathrm{~km} \mathrm{~s}^{-1}$, respectively, for BAL troughs exceeding these limits. It is apparent from Figure 8 that this effect is present for the $v_{\max }$ and less severely for the $v_{\min }$ distributions for $\mathrm{CIV}_{00}, \mathrm{CIV}_{\mathrm{S} 0}$, and $\mathrm{CIV}_{\mathrm{SA}} \mathrm{BAL}$ troughs. We thus compare $v_{\min }$ and $v_{\max }$ distributions using a twosample Peto-Prentice test (PP; e.g., Latta 1981), implemented in the Astronomy Survival Analysis (ASURV; e.g., Lavalley et al. 1992) package, which considers such censoring effects in the samples. The PP test results show that all three $v_{\text {min }}$ distributions are significantly different (at a level of $>99.9 \%$ ) from each other and all three $v_{\max }$ distributions do not show highly significant differences ( $P$ is $30.0 \%$ for the $\mathrm{C} \mathrm{IV}_{00}$ vs. C IV $\mathrm{IV}_{\mathrm{S} 0}$ samples, $35.2 \%$ for the $\mathrm{CIV}_{\mathrm{S} 0}$ vs. $\mathrm{CIV}_{\mathrm{SA}}$ samples, and $75.8 \%$ for the $\mathrm{C}_{I_{0}}$ vs. $\mathrm{C}_{\text {IV }}$ SA samples). We also compare $v_{\min }$ and $v_{\max }$ distributions for the three $\mathrm{C}$ IV groups and find similar results; the consistency of both the PP and AD tests suggests that the truncation of $v_{\min }$ and $v_{\max }$ does not have a significant effect on our main results. Still, we focus our discussion on $v_{\min }$ as per its importance for our comparative assessments (see Section 5) rather than the more affected $v_{\max }$.
The mean $v_{\min }, v_{\max }$, and $\left\langle v_{\text {cent }}\right\rangle$ values are given in Table 9. $\mathrm{CIV}_{00} \mathrm{BAL}$ troughs tend to have higher onset velocities than $\mathrm{CIV}_{\mathrm{S} 0}$ and $\mathrm{CIV}_{\mathrm{SA}}$ troughs. The mean $v_{\text {min }}$ values change by a factor of $\approx 2.5$ from $\mathrm{CIV}_{00}$ to $\mathrm{CIV}_{\text {SA }}$ troughs. Such differences between the $v_{\text {min }}$ distributions for $\mathrm{CIV}_{00}, \mathrm{CIV}_{\mathrm{S} 0}$, and $\mathrm{CIV}_{\mathrm{SA}} \mathrm{BAL}$ troughs are perhaps expected given that the average outflow velocity is higher for weak BAL troughs (e.g., Weymann et al. 1991). Unlike the $v_{\min }$ distributions, the $v_{\max }$ distributions do not show strong apparent differences between the C IV groups.

AD test results show that the $\left\langle v_{\text {cent }}\right\rangle$ distributions are significantly different (at a level of $>99.9 \%$ ) from each other. $\mathrm{CIV}_{\mathrm{SA}} \mathrm{BAL}$ troughs tend to be found at the lowest such velocities, and $\mathrm{CIV}_{00}$ troughs tend to be found at the highest such velocities, although the mean $\left\langle v_{\text {cent }}\right\rangle$ values differ by only $\approx 25 \%$.

\subsection{BAL-Variation Profiles}

We assess the overall differences between C IV BALvariation characteristics by comparing composite variation profiles for $\mathrm{CIV}_{00}, \mathrm{CIV}_{\mathrm{SO}}$, and $\mathrm{CIV}_{\mathrm{SA}}$ troughs. Since BAL-trough widths cover a considerable range for each C IV group (see Figure [7), we divide each group into three bins considering $\Delta v$ values. The bin limits are $\Delta v<3000,3000<\Delta v<5000$, and $\Delta v>5000 \mathrm{~km} \mathrm{~s}^{-1}$ for $\mathrm{C}_{\mathrm{IV}_{00}}$ troughs; $\Delta v<8000,8000<\Delta v<12,000$, and $\Delta v>12,000 \mathrm{~km} \mathrm{~s}^{-1}$ for $\mathrm{CIV}_{\mathrm{S} 0}$ troughs; and $\Delta v<10,000,10,000<\Delta v<14,000$, and $\Delta v>$ 
Table 2

C IV00 Troughs

\begin{tabular}{|c|c|c|c|c|c|c|c|c|}
\hline $\begin{array}{l}\text { Quasar } \\
\text { ID }\end{array}$ & $\begin{array}{c}\mathrm{CIV}_{00} \\
\text { Trough ID }\end{array}$ & $\begin{array}{c}v_{\max } \\
\left(\mathrm{km} \mathrm{s}^{-1}\right)\end{array}$ & $\left(\begin{array}{c}v_{\min } \\
\left(\mathrm{kms}^{-1}\right)\end{array}\right.$ & $\begin{array}{c}\Delta v \\
\left(\mathrm{~km} \mathrm{~s}^{-1}\right)\end{array}$ & $\begin{array}{c}v_{\text {cent }}[1] \\
\left(\mathrm{km} \mathrm{s}^{-1}\right)\end{array}$ & $\begin{array}{c}v_{\text {cent }}[2] \\
\left(\mathrm{km} \mathrm{s}^{-1}\right)\end{array}$ & $d_{\mathrm{BAL}}[1]$ & $\sigma_{d_{\mathrm{BAL}}}[1]$ \\
\hline Q29 & $\mathrm{C} 00-1$ & -10957.2 & -8528.4 & 2428.8 & -9712.9 & -9676.2 & 0.200 & 0.022 \\
\hline Q29 & $\mathrm{C} 00-2$ & -7593.0 & -4686.9 & 2906.1 & -6046.1 & -6072.0 & 0.339 & 0.024 \\
\hline Q34 & $\mathrm{C} 00-3$ & -20000.0 & -17884.3 & 2115.7 & -18892.9 & -18901.7 & 0.124 & 0.009 \\
\hline Q34 & $\mathrm{C} 00-4$ & -17862.3 & -12780.2 & 5082.1 & -15274.8 & -15295.9 & 0.130 & 0.009 \\
\hline Q40 & $\mathrm{C} 00-5$ & -15763.9 & -9381.9 & 6382.0 & -12610.5 & -12497.6 & 0.307 & 0.017 \\
\hline Q41 & $\mathrm{C} 00-6$ & -20000.0 & -16100.5 & 3899.5 & -17974.4 & -17956.4 & 0.348 & 0.020 \\
\hline $\mathrm{Q} 47$ & $\mathrm{C} 00-7$ & -19397.8 & -11712.7 & 7685.1 & -15810.3 & -15687.3 & 0.368 & 0.015 \\
\hline Q47 & $\mathrm{C} 00-8$ & -11537.6 & -8089.2 & 3448.4 & -9811.0 & -9823.4 & 0.329 & 0.023 \\
\hline Q51 & C00-9 & -9963.7 & -7962.3 & 2001.5 & -8946.8 & -8954.2 & 0.144 & 0.021 \\
\hline Q57 & C00-10 & -20000.0 & -4959.0 & 15041.0 & -12291.8 & -12402.4 & 0.189 & 0.006 \\
\hline
\end{tabular}

\begin{tabular}{|c|c|c|c|c|c|c|c|c|c|}
\hline$d_{\mathrm{BAL}}[2]$ & $\sigma_{d_{\mathrm{BAL}}}[2]$ & $\begin{array}{r}\mathrm{EW}[1] \\
(\AA)\end{array}$ & $\begin{array}{r}\sigma_{\mathrm{EW}}[1] \\
(\AA)\end{array}$ & $\begin{array}{r}\mathrm{EW}[2] \\
(\AA)\end{array}$ & $\begin{array}{r}\sigma_{\mathrm{EW}}[2] \\
(\AA)\end{array}$ & $\begin{array}{r}\Delta \mathrm{EW} \\
(\AA)\end{array}$ & $\begin{array}{r}\sigma_{\Delta E W} \\
(\AA)\end{array}$ & $\frac{\Delta \mathrm{EW}}{\langle\mathrm{EW}\rangle}$ & $\sigma_{\frac{\Delta \mathrm{EW}}{\langle\mathrm{EW}\rangle}}$ \\
\hline 0.147 & 0.015 & 2.37 & 0.19 & 1.65 & 0.18 & -0.72 & 0.26 & -0.36 & 0.18 \\
\hline 0.311 & 0.020 & 5.10 & 0.20 & 4.71 & 0.17 & -0.39 & 0.26 & -0.08 & 0.08 \\
\hline 0.133 & 0.005 & 1.27 & 0.16 & 1.42 & 0.05 & 0.15 & 0.17 & 0.11 & 0.16 \\
\hline 0.145 & 0.005 & 3.18 & 0.25 & 3.67 & 0.08 & 0.49 & 0.26 & 0.14 & 0.10 \\
\hline 0.294 & 0.013 & 9.49 & 0.50 & 9.37 & 0.21 & -0.12 & 0.54 & -0.01 & 0.07 \\
\hline 0.279 & 0.015 & 6.76 & 0.22 & 5.45 & 0.18 & -1.30 & 0.28 & -0.21 & 0.06 \\
\hline 0.118 & 0.010 & 13.77 & 0.61 & 3.70 & 0.24 & -10.08 & 0.66 & -1.15 & 0.07 \\
\hline 0.189 & 0.016 & 5.68 & 0.35 & 3.21 & 0.13 & -2.47 & 0.37 & -0.56 & 0.09 \\
\hline 0.394 & 0.036 & 1.23 & 0.26 & 4.10 & 0.17 & 2.87 & 0.31 & 1.08 & 0.18 \\
\hline 0.207 & 0.004 & 13.86 & 0.83 & 15.56 & 0.62 & 1.70 & 1.03 & 0.12 & 0.10 \\
\hline
\end{tabular}

Notes. Throughout this table [1] indicates the first-epoch spectra and [2] indicates the second-epoch spectra.

(This table is available in its entirety in a machine-readable form in the online journal. A portion is shown here for guidance regarding its form and content.)

$14,000 \mathrm{~km} \mathrm{~s}^{-1}$ for $\mathrm{C}$ IV SA troughs.

First, we calculate the absolute value of the depth variation between the SDSS and BOSS spectra for each data point of each trough as a function of outflow velocity, $v_{\mathrm{t}}$. Second, we calculate the mean absolute depth variation for BAL troughs in each bin by interpolating the data points for given $v_{\mathrm{t}}$ values. Figure 9 displays the mean composites of depth-variation profiles for $\mathrm{C}_{\mathrm{IV}} 00$ troughs with $3000<\Delta v<5000 \mathrm{~km} \mathrm{~s}^{-1}$, $\mathrm{C}$ IV $0_{\mathrm{S} 0}$ troughs with $8000<\Delta v<12,000 \mathrm{~km} \mathrm{~s}^{-1}$, and $\mathrm{CIV}_{\mathrm{SA}}$ troughs with $10000<\Delta v<14,000 \mathrm{~km} \mathrm{~s}^{-1}$. We find qualitatively consistent results when computing median composites instead of mean composites. Figure 9 also presents the mean composite for absolute fractional variations which is the variation of depth between SDSS and BOSS divided by the average depth as a function of $v_{\mathrm{t}}$.

The composite variation profiles demonstrate that the level of variation generally tends to be smaller for the lower velocity portions of C IV troughs. Note that in each panel of Figure 9, the trough number statistics are limited for the highest velocity end. Figure 9 (left panels) shows that the lower velocity portions of $\mathrm{C}_{\mathrm{IV}_{\mathrm{S} 0}}$ troughs (up to $\approx 30 \%$ of the trough width) tend to be less variable than the higher velocity portions, and these less variable portions usually correspond to regions where the Si IV BAL troughs are strongest (see Figure 5). The variation profile for $\mathrm{CIV}_{\mathrm{SA}}$ troughs is strongly different from the variation profiles for $\mathrm{CIV}_{00}$ and $\mathrm{CIV}_{\mathrm{S} 0}$ troughs. For $\mathrm{CIV}_{\mathrm{SA}}$ troughs, the level of variability is notably low for up to $\approx 80 \%$ of the trough width; this result is highly statistically significant, considering the number of independent spectral data points and their error bars. Figure 5 demonstrates that these lower velocity portions of $\mathrm{C}_{\text {IV }}$ A troughs correspond to regions where Al III BAL troughs are generally found.

Composite fractional-variation profiles further highlight the differences between the variation characteristics of $\mathrm{CIV}_{00}, \mathrm{CIV}_{\mathrm{S} 0}$, and $\mathrm{CIV}_{\mathrm{SA}} \mathrm{BAL}$ troughs. These arguably allow the best possible comparison of overall variability between these $\mathrm{C}$ IV groups, since they account for the significantly different strengths of $\mathrm{CIV}_{00}, \mathrm{C}_{\mathrm{IV}_{\mathrm{S} 0}}$, and $\mathrm{CIV}_{\mathrm{SA}}$ troughs (see Section 4.2). Overall, we find that $\mathrm{CIV}_{00}$ troughs tend to be significantly more fractionally variable than both $\mathrm{CIV}_{\mathrm{S} 0}$ and $\mathrm{CIV}_{\mathrm{SA}}$ troughs, and likewise $\mathrm{CIV}_{\mathrm{S} 0}$ troughs tend to be somewhat more fractionally variable than $\mathrm{C}_{\mathrm{IV}_{\mathrm{SA}}}$ troughs. Variability is again smallest in the lower velocity portions of $\mathrm{C} \mathrm{IV}_{\mathrm{S} 0}$ and $\mathrm{CIV}_{\text {SA }}$ troughs. However, this behavior is not apparent for $\mathrm{CIV}_{00}$ troughs.

\subsection{BAL EW Variability}

4.5.1. EW Variability as a Function of $\langle\mathrm{EW}\rangle$ 
Table 3

C IV So Troughs

\begin{tabular}{|c|c|c|c|c|c|c|c|c|}
\hline $\begin{array}{l}\text { Quasar } \\
\text { ID }\end{array}$ & $\begin{array}{c}\text { CIVSo } \\
\text { Trough ID }\end{array}$ & $\begin{array}{c}v_{\max } \\
\left(\mathrm{km} \mathrm{s}^{-1}\right)\end{array}$ & $\begin{array}{c}v_{\min } \\
\left(\mathrm{km} \mathrm{s}^{-1}\right)\end{array}$ & $\begin{array}{c}\Delta v \\
\left(\mathrm{~km} \mathrm{~s}^{-1}\right)\end{array}$ & $\begin{array}{c}v_{\text {cent }}[1] \\
\left(\mathrm{km} \mathrm{s}^{-1}\right)\end{array}$ & $\begin{array}{c}v_{\text {cent }}[2] \\
\left(\mathrm{km} \mathrm{s}^{-1}\right)\end{array}$ & $d_{\mathrm{BAL}}[1]$ & $\sigma_{d_{\mathrm{BAL}}}[1]$ \\
\hline Q3 & CSO-1 & -10477.0 & -5841.4 & 4635.7 & -8427.4 & -8285.0 & 0.491 & 0.032 \\
\hline Q4 & CSO-2 & -12444.7 & -4858.5 & 7586.2 & -9558.5 & -9262.8 & 0.697 & 0.025 \\
\hline Q10 & CSO-3 & -15491.2 & -3000.0 & 12491.2 & -10869.6 & -10629.1 & 0.531 & 0.024 \\
\hline Q11 & $\mathrm{CSO}-4$ & -11596.2 & -5638.0 & 5958.2 & -8516.9 & -8806.4 & 0.217 & 0.010 \\
\hline Q12 & CSO-5 & -10921.6 & -3000.0 & 7921.6 & -8088.0 & -8031.0 & 0.648 & 0.023 \\
\hline
\end{tabular}

\begin{tabular}{|c|c|c|c|c|c|c|c|c|c|}
\hline$d_{\mathrm{BAL}}[2]$ & $\sigma_{d_{\mathrm{BAL}}}[2]$ & $\begin{array}{r}\mathrm{EW}[1] \\
(\AA)\end{array}$ & $\begin{array}{r}\sigma_{\mathrm{EW}}[1] \\
(\AA)\end{array}$ & $\begin{array}{r}\mathrm{EW}[2] \\
(\AA)\end{array}$ & $\begin{array}{r}\sigma_{\mathrm{EW}}[2] \\
(\AA)\end{array}$ & $\begin{array}{r}\Delta \mathrm{EW} \\
(\AA)\end{array}$ & $\begin{array}{r}\sigma_{\Delta E W} \\
(\AA)\end{array}$ & $\frac{\Delta \mathrm{EW}}{\langle\mathrm{EW}\rangle}$ & $\sigma_{\frac{\Delta \mathrm{EW}}{\langle\mathrm{EW}\rangle}}$ \\
\hline 0.442 & 0.029 & 11.51 & 0.56 & 10.29 & 0.15 & -1.22 & 0.58 & -0.11 & 0.06 \\
\hline 0.744 & 0.023 & 26.86 & 0.20 & 28.43 & 0.10 & 1.56 & 0.22 & 0.06 & 0.01 \\
\hline 0.543 & 0.021 & 31.37 & 0.92 & 35.28 & 0.73 & 3.91 & 1.18 & 0.12 & 0.05 \\
\hline 0.253 & 0.016 & 6.63 & 0.15 & 7.73 & 0.19 & 1.10 & 0.24 & 0.15 & 0.05 \\
\hline 0.593 & 0.024 & 27.95 & 0.32 & 25.64 & 0.22 & -2.31 & 0.39 & -0.09 & 0.02 \\
\hline
\end{tabular}

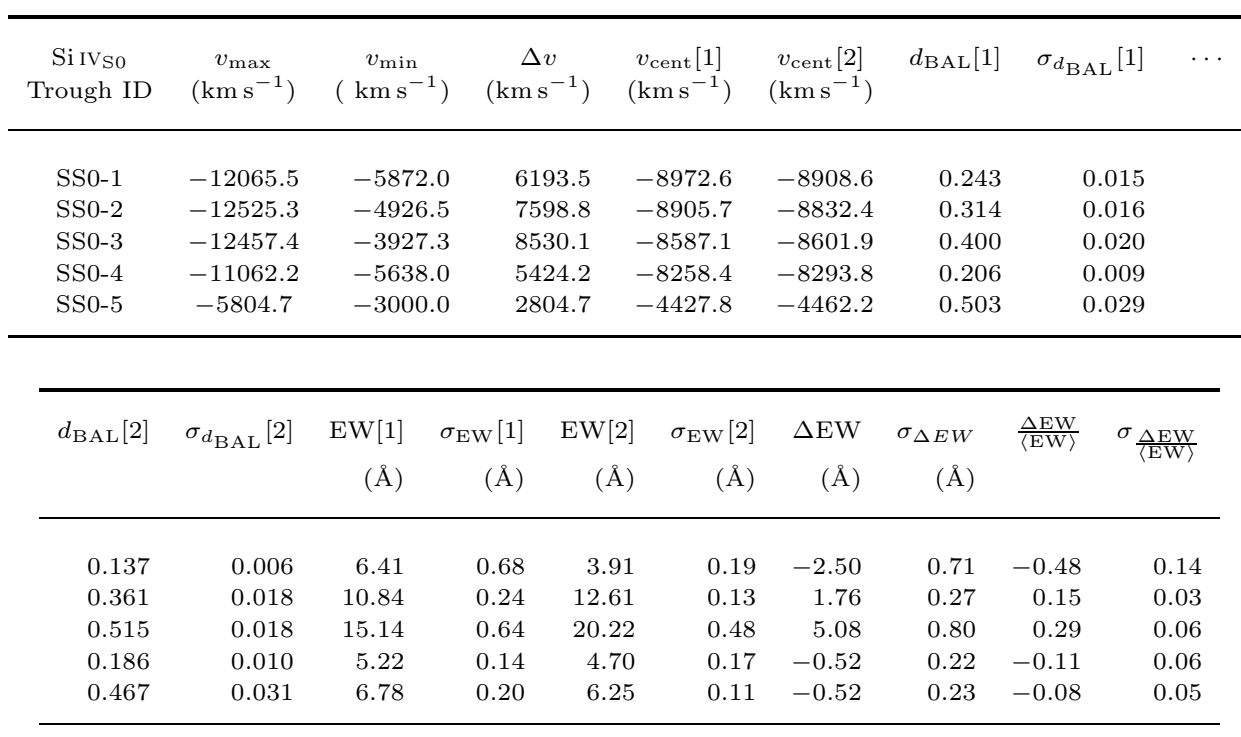

Notes. Throughout this table [1] indicates the first-epoch spectra and [2] indicates the second-epoch spectra.

(This table is available in its entirety in a machine-readable form in the online journal. A portion is shown here for guidance regarding its form and content.)

In this section, we investigate the characteristics of BAL EW variability assessing the differences between $\mathrm{C} I V_{00}, \mathrm{CIV}_{\mathrm{S} 0}$, and $\mathrm{CIV}_{\mathrm{SA}}$ troughs. Figure 10 presents the EW variation, $\triangle \mathrm{EW}$, between the two-epoch spectra as a function of average $\mathrm{EW},\langle\mathrm{EW}\rangle$. For comparison, the standard-deviation curves for these three C IV groups are also given; the standard-deviation curves are calculated using a sliding window where each window contains $10 \%$ of the total number of BAL troughs found in a given group (i.e., 11 for $\mathrm{CIV}_{00}, 25$ for $\mathrm{C} \mathrm{IV}_{\mathrm{S} 0}$, and 10 for $\left.\mathrm{CIV}_{\mathrm{SA}}\right)$. We statistically remove the mean $\mathrm{EW}$ error in each window from the standard deviation, so the dispersion shown in Figure 10 refers to the intrinsic dispersion.
Consistent with Section 4.5 of Filiz Ak et al. (2013), we find that the standard deviation of $\triangle \mathrm{EW}$ generally increases with increasing $\langle\mathrm{EW}\rangle$ for all $\mathrm{C}$ IV groups. Given that such a trend exists for all C IV groups, a proper intergroup comparison requires consideration of $\mathrm{EW}$ variation for troughs with similar $\langle\mathrm{EW}\rangle$ values. The $\mathrm{CIV}_{\mathrm{S} 0} \Delta \mathrm{EW}$ spread appears less than that for $\mathrm{C}_{\mathrm{IV}_{00}}$ BALs with similar $\langle\mathrm{EW}\rangle$ values (i.e., $7<\langle\mathrm{EW}\rangle<10 \AA$ ). Similarly, the $\mathrm{CIV}_{\text {SA }} \Delta \mathrm{EW}$ spread appears less than that for $\mathrm{CIV}_{\mathrm{S} 0}$ BALs for $12<\langle\mathrm{EW}\rangle<35 \AA$. These results suggest that $\mathrm{C}$ IV EW variation has a dependence upon the existence of absorption lines from Si IV and Al III transitions.

In Figure 11, we show the fractional EW variation, $\Delta \mathrm{EW} /\langle\mathrm{EW}\rangle$, as a function of $\langle\mathrm{EW}\rangle$ for $\mathrm{C} \mathrm{IV}_{00}, \mathrm{C}_{\mathrm{IV}} \mathrm{SO}$, 
Table 4

C IV SA Troughs

\begin{tabular}{ccccccccc}
\hline $\begin{array}{c}\text { Quasar } \\
\text { ID }\end{array}$ & $\begin{array}{c}\text { CIVSA } \\
\text { Trough ID }\end{array}$ & $\begin{array}{c}v_{\max } \\
\left(\mathrm{km} \mathrm{s}^{-1}\right)\end{array}$ & $\begin{array}{c}v_{\min } \\
\left(\mathrm{km} \mathrm{s}^{-1}\right)\end{array}$ & $\begin{array}{c}\Delta v \\
\left(\mathrm{~km} \mathrm{~s}^{-1}\right)\end{array}$ & $\begin{array}{c}v_{\text {cent }}[1] \\
\left(\mathrm{km} \mathrm{s}^{-1}\right)\end{array}$ & $\begin{array}{c}v_{\text {cent }}[2] \\
\left(\mathrm{km} \mathrm{s}^{-1}\right)\end{array}$ & $d_{\mathrm{BAL}}[1]$ & $\sigma_{d_{\mathrm{BAL}}[1]}$ \\
\hline & & & & & & & \\
Q1 & CSA-1 & -17205.7 & -3487.4 & 13718.4 & -10850.0 & -10770.0 & 0.521 & 0.018 \\
Q5 & CSA-2 & -20000.0 & -4805.6 & 15194.4 & -13846.9 & -13939.7 & 0.755 & 0.011 \\
Q8 & CSA-3 & -20000.0 & -3000.0 & 17000.0 & -11866.5 & -11868.8 & 0.450 & 0.015 \\
Q20 & CSA-4 & -20000.0 & -6837.8 & 13162.2 & -13057.2 & -12687.1 & 0.607 & 0.014 \\
Q28 & CSA-5 & -20000.0 & -7283.0 & 12717.0 & -14376.7 & -14356.4 & 0.291 & 0.014 \\
\hline
\end{tabular}

\begin{tabular}{|c|c|c|c|c|c|c|c|c|c|}
\hline$d_{\mathrm{BAL}}[2]$ & $\sigma_{d_{\mathrm{BAL}}}[2]$ & $\begin{array}{r}\mathrm{EW}[1] \\
(\AA)\end{array}$ & $\begin{array}{r}\sigma_{\mathrm{EW}}[1] \\
(\AA)\end{array}$ & $\begin{array}{r}\mathrm{EW}[2] \\
(\AA)\end{array}$ & $\begin{array}{r}\sigma_{\mathrm{EW}}[2] \\
(\AA)\end{array}$ & $\begin{array}{r}\Delta \mathrm{EW} \\
(\AA)\end{array}$ & $\begin{array}{r}\sigma_{\Delta E W} \\
(\AA)\end{array}$ & $\frac{\Delta \mathrm{EW}}{\langle\mathrm{EW}\rangle}$ & $\sigma_{\frac{\Delta \mathrm{EW}}{\langle\mathrm{EW}\rangle}}$ \\
\hline 0.527 & 0.018 & 33.47 & 0.80 & 36.51 & 0.46 & 3.04 & 0.93 & 0.09 & 0.04 \\
\hline 0.752 & 0.011 & 58.06 & 0.84 & 57.61 & 0.45 & -0.45 & 0.95 & -0.01 & 0.02 \\
\hline 0.532 & 0.014 & 38.15 & 1.08 & 45.28 & 0.57 & 7.13 & 1.22 & 0.17 & 0.04 \\
\hline 0.634 & 0.013 & 39.57 & 0.78 & 41.52 & 0.73 & 1.95 & 1.07 & 0.05 & 0.04 \\
\hline 0.306 & 0.015 & 19.46 & 0.42 & 20.19 & 0.22 & 0.73 & 0.47 & 0.04 & 0.03 \\
\hline
\end{tabular}

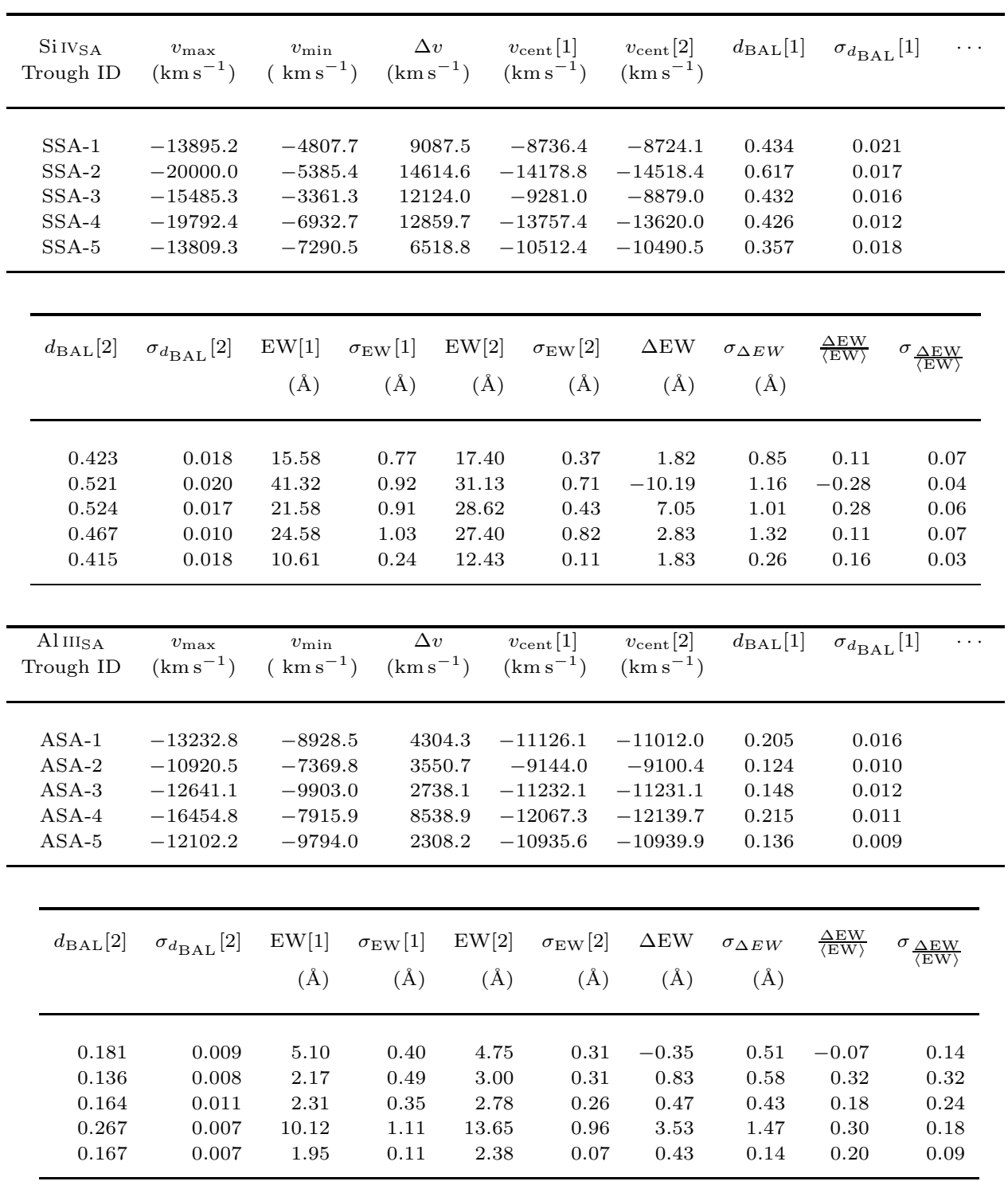

Notes. Throughout this table [1] indicates the first-epoch spectra and [2] indicates the second-epoch spectra.

(This table is available in its entirety in a machine-readable form in the online journal. A portion is shown here for guidance regarding its form and content.) 
Table 5

$\mathrm{C}_{\mathrm{IV}} \mathrm{s} 0$ Troughs

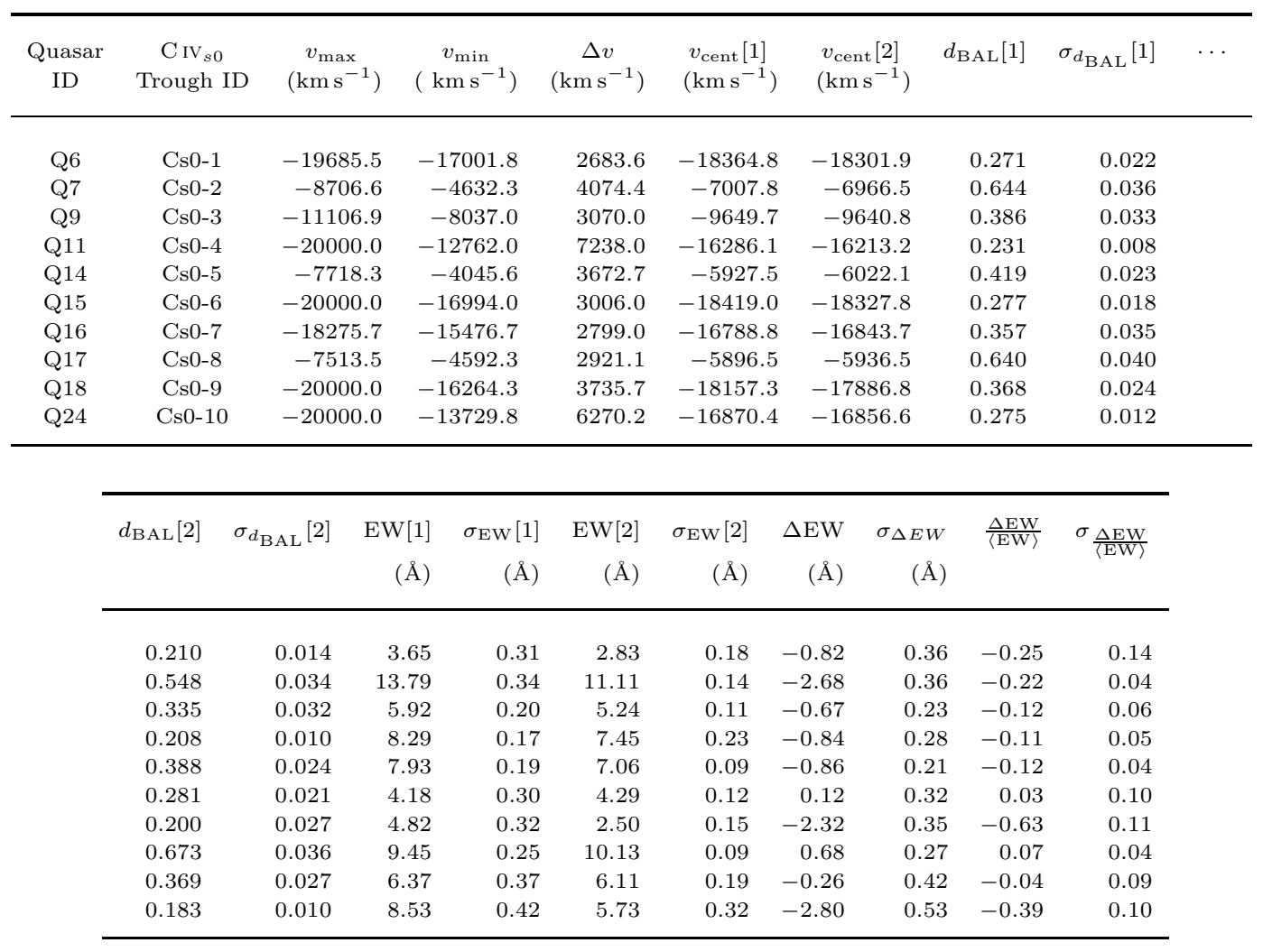

Notes. Throughout this table [1] indicates the first-epoch spectra and [2] indicates the second-epoch spectra.

(This table is available in its entirety in a machine-readable form in the online journal. A portion is shown here for guidance regarding its form and content.) 
Table 6

C IV sa Troughs

\begin{tabular}{|c|c|c|c|c|c|c|c|c|}
\hline $\begin{array}{l}\text { Quasar } \\
\text { ID }\end{array}$ & $\begin{array}{c}\mathrm{CIV}_{s a} \\
\text { Trough ID }\end{array}$ & $\begin{array}{c}v_{\max } \\
\left(\mathrm{km} \mathrm{s}^{-1}\right)\end{array}$ & $\begin{array}{c}v_{\min } \\
\left(\mathrm{km} \mathrm{s}^{-1}\right)\end{array}$ & $\begin{array}{c}\Delta v \\
\left(\mathrm{~km} \mathrm{~s}^{-1}\right)\end{array}$ & $\begin{array}{c}v_{\text {cent }}[1] \\
\left(\mathrm{km} \mathrm{s}^{-1}\right)\end{array}$ & $\begin{array}{c}v_{\text {cent }}[2] \\
\left(\mathrm{km} \mathrm{s}^{-1}\right)\end{array}$ & $d_{\mathrm{BAL}}[1]$ & $\sigma_{d_{\mathrm{BAL}}}[1]$ \\
\hline Q68 & Csa-1 & -16520.3 & -7967.2 & 8553.2 & -12538.7 & -12413.4 & 0.470 & 0.019 \\
\hline Q74 & Csa-2 & -12104.4 & -5549.0 & 6555.4 & -8424.5 & -8381.3 & 0.542 & 0.025 \\
\hline Q92 & Csa-3 & -15812.8 & -7274.5 & 8538.3 & -11413.4 & -11523.5 & 0.307 & 0.018 \\
\hline Q95 & Csa-4 & -20000.0 & -7477.7 & 12522.3 & -14343.8 & -13767.6 & 0.408 & 0.015 \\
\hline Q102 & Csa-5 & -15573.0 & -9850.9 & 5722.1 & -12641.0 & -12533.8 & 0.327 & 0.017 \\
\hline Q121 & Csa-6 & -16532.5 & -7118.1 & 9414.4 & -11798.3 & -11969.2 & 0.335 & 0.016 \\
\hline Q122 & Csa-7 & -12716.2 & -4706.0 & 8010.1 & -8953.3 & -9023.5 & 0.310 & 0.017 \\
\hline Q192 & Csa-8 & -16109.3 & -11388.8 & 4720.5 & -13675.9 & -13745.2 & 0.098 & 0.009 \\
\hline Q217 & Csa-9 & -6388.4 & -4238.7 & 2149.7 & -5038.8 & -5092.9 & 0.711 & 0.053 \\
\hline Q240 & Csa-10 & -11158.8 & -7751.6 & 3407.3 & -9243.6 & -9357.1 & 0.415 & 0.036 \\
\hline
\end{tabular}

\begin{tabular}{|c|c|c|c|c|c|c|c|c|c|}
\hline$d_{\mathrm{BAL}}[2]$ & $\sigma_{d_{\mathrm{BAL}}}[2]$ & $\begin{array}{r}\mathrm{EW}[1] \\
(\AA)\end{array}$ & $\begin{array}{r}\sigma_{\mathrm{EW}}[1] \\
(\AA)\end{array}$ & $\begin{array}{r}\mathrm{EW}[2] \\
(\AA)\end{array}$ & $\begin{array}{r}\sigma_{\mathrm{EW}}[2] \\
(\AA)\end{array}$ & $\begin{array}{r}\Delta \mathrm{EW} \\
(\AA)\end{array}$ & $\begin{array}{r}\sigma_{\Delta E W} \\
(\AA)\end{array}$ & $\frac{\Delta \mathrm{EW}}{\langle\mathrm{EW}\rangle}$ & $\sigma_{\frac{\Delta \mathrm{EW}}{\langle\mathrm{EW}\rangle}}$ \\
\hline 0.225 & 0.015 & 20.15 & 0.82 & 9.04 & 0.41 & -11.10 & 0.91 & -0.76 & 0.07 \\
\hline 0.317 & 0.024 & 18.23 & 0.53 & 8.10 & 0.46 & -10.12 & 0.71 & -0.77 & 0.07 \\
\hline 0.348 & 0.016 & 12.82 & 0.96 & 15.08 & 0.57 & 2.26 & 1.12 & 0.16 & 0.11 \\
\hline 0.152 & 0.006 & 25.10 & 1.20 & 9.16 & 0.59 & -15.94 & 1.34 & -0.93 & 0.09 \\
\hline 0.386 & 0.019 & 8.51 & 0.66 & 10.35 & 0.43 & 1.84 & 0.79 & 0.20 & 0.12 \\
\hline 0.295 & 0.013 & 13.48 & 0.76 & 13.89 & 0.44 & 0.41 & 0.87 & 0.03 & 0.09 \\
\hline 0.339 & 0.016 & 12.08 & 0.95 & 13.79 & 0.27 & 1.71 & 0.99 & 0.13 & 0.10 \\
\hline 0.200 & 0.008 & 1.52 & 0.43 & 4.71 & 0.51 & 3.19 & 0.66 & 1.02 & 0.29 \\
\hline 0.626 & 0.056 & 8.15 & 0.25 & 6.81 & 0.11 & -1.35 & 0.27 & -0.18 & 0.05 \\
\hline 0.225 & 0.022 & 7.18 & 0.22 & 3.42 & 0.13 & -3.76 & 0.25 & -0.71 & 0.06 \\
\hline
\end{tabular}

Notes. Throughout this table [1] indicates the first-epoch spectra and [2] indicates the second-epoch spectra.

(This table is available in its entirety in a machine-readable form in the online journal. A portion is shown here for guidance regarding its form and content.)

and IV $_{\text {SA }}$ troughs. Similarly to Figure 10, solid curves represent the sliding-window standard deviations. The overall trend in $\Delta \mathrm{EW} /\langle\mathrm{EW}\rangle$ vs. $\langle\mathrm{EW}\rangle$ appears mainly due to the increase of $\langle\mathrm{EW}\rangle$. The curves indicate that the spread of $\Delta \mathrm{EW} /\langle\mathrm{EW}\rangle$ for $\mathrm{CIV}_{00}$ is larger than that for $\mathrm{C}_{\mathrm{IV}} \mathrm{S}$, and the $\Delta \mathrm{EW} /\langle\mathrm{EW}\rangle$ spread for $\mathrm{CIV}_{\mathrm{S} 0}$ is larger than that for $\mathrm{CIV}_{\mathrm{SA}}$ in matched $\langle\mathrm{EW}\rangle$ ranges.

Previous BAL-variability studies (Gibson et al. 2008, 2010; Capellupo et al. 2011; Filiz Ak et al. 2013) have demonstrated that BAL EW variability increases with increasing timescale. However, the trends in Figures 10 and 11 are not caused or amplified by this effect; the testing in Section 3 showed that the timescale distributions for $\mathrm{CIV}_{00}, \mathrm{C}_{\mathrm{IV}} \mathrm{S}$, and $\mathrm{CIV}_{\mathrm{SA}}$ troughs do not have any significant differences from each other (see Figure 4).

Considering that the $\langle\mathrm{EW}\rangle$ distributions are significantly different between the three C IV groups (see Figure 6) and that there are strong correlations between $\Delta \mathrm{EW}$ or $|\Delta \mathrm{EW} /\langle\mathrm{EW}\rangle|$ and $\langle\mathrm{EW}\rangle$ for all C IV troughs (also see Section 4.5 of Filiz Ak et al. 2013), we investigate the EW variation distributions of these three groups using matched samples of troughs with similar EWs.

\subsubsection{BAL-Trough Samples with Matching EWs}

We investigated the EW-variation characteristics of $\mathrm{CIV}_{00}$ vs. $\mathrm{CIV}_{\mathrm{S} 0}$ and $\mathrm{CIV}_{\mathrm{S} 0}$ vs. $\mathrm{CIV}_{\mathrm{SA}}$ troughs using matched samples. These matched samples contain the same number of BAL troughs from both groups matched by first-epoch $\mathrm{EW}, \mathrm{EW}_{1}$. Requiring similar $\mathrm{EW}_{1}$ for each trough pair allows comparison of variation behaviors for $\mathrm{C}$ IV groups having similar initial conditions. We first randomly select a $\mathrm{CIV}_{00}$ trough for each $\mathrm{C}_{\mathrm{IV}} \mathrm{So}$ trough such that their EWs are in agreement to within $1 \sigma$. Due to significant differences in the EW distributions, we cannot match all data points of a group. In the matching procedure, we sample any given BAL trough only once. Second, we applied a two-sample AD test to compare the $\triangle \mathrm{EW}$ distributions. We repeated the matching and $\mathrm{AD}$ testing 1000 times and found the median results given in Table 10 .

Comparing the $\Delta \mathrm{EW}$ distributions of $\mathrm{C} \mathrm{IV}_{00}$ and $\mathrm{C} \mathrm{IV}_{\mathrm{SO}}$ BAL troughs with matched EWs (where each distribution has 40 troughs), we found that the two distributions significantly $(P>99.9 \%)$ differ from each other. Similarly, we repeat the matching for $\mathrm{C}_{\mathrm{IV}_{\mathrm{S} 0}}$ and $\mathrm{CIV}_{\mathrm{SA}}$ troughs. The test results for the comparison between the $\triangle \mathrm{EW}$ distributions of $\mathrm{CIV}_{\mathrm{S} 0}$ and $\mathrm{CIV}_{\mathrm{SA}}$ troughs with similar EWs (where each has 65 troughs) show that the two distributions differ with a significance of $97.8 \%$. Figure 12 presents the $\Delta \mathrm{EW}$ distributions for $\mathrm{CIV}_{00}$ and $\mathrm{CIV}_{\text {S0 }}$ BAL troughs with matched EWs in the left panel, and $\mathrm{CIV}_{\mathrm{S} 0}$ and $\mathrm{CIV}_{\mathrm{SA}}$ BAL troughs with matched EWs in the right panel. 
Table 7

C IV $_{\mathrm{Sa}}$ Troughs

\begin{tabular}{|c|c|c|c|c|c|c|c|c|}
\hline $\begin{array}{l}\text { Quasar } \\
\text { ID }\end{array}$ & $\begin{array}{c}\text { CIV }_{S a} \\
\text { Trough ID }\end{array}$ & $\begin{array}{c}v_{\max } \\
\left(\mathrm{km} \mathrm{s}^{-1}\right)\end{array}$ & $\begin{array}{c}v_{\min } \\
\left(\mathrm{km} \mathrm{s}^{-1}\right)\end{array}$ & $\begin{array}{c}\Delta v \\
\left(\mathrm{~km} \mathrm{~s}^{-1}\right)\end{array}$ & $\begin{array}{c}v_{\text {cent }}[1] \\
\left(\mathrm{km} \mathrm{s}^{-1}\right)\end{array}$ & $\begin{array}{c}v_{\text {cent }}[2] \\
\left(\mathrm{km} \mathrm{s}^{-1}\right)\end{array}$ & $d_{\mathrm{BAL}}[1]$ & $\sigma_{d_{\mathrm{BAL}}}[1]$ \\
\hline Q2 & CSa-1 & -20000.0 & -7997.7 & 12002.3 & -14588.0 & -14972.5 & 0.409 & 0.018 \\
\hline Q19 & CSa-2 & -9532.0 & -3000.0 & 6532.0 & -7219.7 & -7519.0 & 0.537 & 0.026 \\
\hline Q21 & CSa-3 & -20000.0 & -7452.5 & 12547.5 & -14198.9 & -14177.9 & 0.698 & 0.015 \\
\hline Q33 & $\mathrm{CSa}-4$ & -20000.0 & -6346.1 & 13653.9 & -14370.2 & -14044.3 & 0.448 & 0.020 \\
\hline Q39 & CSa-5 & -10772.3 & -3000.0 & 7772.3 & -8305.6 & -8191.4 & 0.592 & 0.026 \\
\hline Q43 & CSa-6 & -20000.0 & -7389.2 & 12610.8 & -15426.7 & -15715.8 & 0.548 & 0.021 \\
\hline Q45 & CSa-7 & -5099.9 & -3000.0 & 2099.9 & -4180.0 & -4273.3 & 0.473 & 0.032 \\
\hline Q46 & CSa-8 & -10647.4 & -4855.0 & 5792.4 & -8520.5 & -8436.7 & 0.791 & 0.028 \\
\hline Q49 & CSa-9 & -12874.5 & -7052.6 & 5821.9 & -9976.5 & -10007.5 & 0.404 & 0.022 \\
\hline Q50 & CSa-10 & -6340.5 & -3018.0 & 3322.5 & -4813.8 & -5072.8 & 0.705 & 0.039 \\
\hline
\end{tabular}

\begin{tabular}{|c|c|c|c|c|c|c|c|c|c|}
\hline$d_{\mathrm{BAL}}[2]$ & $\sigma_{d_{\mathrm{BAL}}}[2]$ & $\begin{array}{r}\mathrm{EW}[1] \\
(\AA)\end{array}$ & $\begin{array}{r}\sigma_{\mathrm{EW}}[1] \\
(\AA)\end{array}$ & $\begin{array}{r}\mathrm{EW}[2] \\
(\AA)\end{array}$ & $\begin{array}{r}\sigma_{\mathrm{EW}}[2] \\
(\AA)\end{array}$ & $\begin{array}{r}\Delta \mathrm{EW} \\
(\AA)\end{array}$ & $\begin{array}{r}\sigma_{\Delta E W} \\
(\AA)\end{array}$ & $\frac{\Delta \mathrm{EW}}{\langle\mathrm{EW}\rangle}$ & $\sigma_{\frac{\Delta \mathrm{EW}}{\langle\mathrm{EW}\rangle}}$ \\
\hline 0.406 & 0.018 & 21.82 & 1.52 & 24.36 & 0.51 & 2.54 & 1.60 & 0.11 & 0.09 \\
\hline 0.591 & 0.029 & 19.20 & 0.45 & 20.23 & 0.31 & 1.03 & 0.54 & 0.05 & 0.04 \\
\hline 0.703 & 0.015 & 43.90 & 0.15 & 44.02 & 0.15 & 0.11 & 0.22 & 0.00 & 0.01 \\
\hline 0.498 & 0.016 & 29.36 & 1.19 & 33.83 & 0.63 & 4.47 & 1.35 & 0.14 & 0.06 \\
\hline 0.641 & 0.022 & 24.24 & 0.60 & 25.89 & 0.77 & 1.64 & 0.97 & 0.07 & 0.05 \\
\hline 0.574 & 0.023 & 34.78 & 1.03 & 36.19 & 0.33 & 1.41 & 1.08 & 0.04 & 0.04 \\
\hline 0.653 & 0.034 & 5.24 & 0.16 & 7.42 & 0.08 & 2.18 & 0.18 & 0.34 & 0.04 \\
\hline 0.788 & 0.028 & 23.43 & 0.11 & 23.31 & 0.12 & -0.12 & 0.17 & 0.00 & 0.01 \\
\hline 0.413 & 0.018 & 11.73 & 0.65 & 12.15 & 0.28 & 0.42 & 0.71 & 0.03 & 0.08 \\
\hline 0.703 & 0.045 & 12.35 & 0.37 & 11.87 & 0.07 & -0.48 & 0.38 & -0.04 & 0.04 \\
\hline
\end{tabular}

Notes. Throughout this table [1] indicates the first-epoch spectra and [2] indicates the second-epoch spectra.

(This table is available in its entirety in a machine-readable form in the online journal. A portion is shown here for guidance regarding its form and content.)

As given in Table 10, the mean of $|\Delta \mathrm{EW}|$ for $\mathrm{C}_{\mathrm{IV}} \mathrm{D}_{00}$ troughs is $\approx 1.3$ times larger than that for matched $\mathrm{C}_{\mathrm{IV}} \mathrm{So}$ troughs. Likewise, the mean of $|\Delta \mathrm{EW}|$ for $\mathrm{CIV}_{\mathrm{S} 0}$ troughs is $\approx 1.8$ times larger than that for matched $\mathrm{C}_{\mathrm{IV} \text { SA }}$ troughs. The standard deviation of the $\Delta \mathrm{EW}$ distribution for $\mathrm{CIV}_{00}$ troughs is $\approx 1.1$ times larger than that for matched $\mathrm{CIV}_{\mathrm{S} 0}$ troughs, and for $\mathrm{CIV}_{\mathrm{S} 0}$ troughs is $\approx 1.7$ times larger than that for matched $\mathrm{C}_{\mathrm{SV}} \mathrm{SA}$ troughs.

Although the matched $\mathrm{CIV}_{\mathrm{SO}}$ and $\mathrm{CIV}_{\mathrm{SA}} \mathrm{BAL}$ troughs have both a larger sample size (65) and difference between the $\sigma_{\Delta \mathrm{EW}}$ values $(5.16-3.09=2.07)$ than the matched $\mathrm{CIV}_{00}$ and $\mathrm{CIV}_{\mathrm{S} 0}$ troughs, the $\mathrm{AD}$-test results indicate a more significant overall difference for the $\Delta \mathrm{EW}$ distributions of $\mathrm{CIV}_{00}$ and $\mathrm{CIV}_{\mathrm{S} 0}$ troughs. This result is mainly due to a more significant difference between the mean $\Delta \mathrm{EW}$ values of matching $\mathrm{CIV}_{00}$ and $\mathrm{CIV}_{\mathrm{S} 0}$ troughs $(3.6 \sigma)$ than that for matching $\mathrm{CIV}_{\mathrm{S} 0}$ and $\mathrm{CIV}_{\mathrm{SA}}$ troughs $(1.9 \sigma)$. Another notable result of these comparisons is that the means of the $\Delta \mathrm{EW}$ distributions for both the $\mathrm{CIV}_{00}$ and $\mathrm{CIV}_{\mathrm{S} 0}$ troughs with similar EWs differ from zero at more than $2 \sigma:-1.13 \pm 0.47 \AA$ and $1.33 \pm 0.49 \AA$, respectively. Moreover, the means of the $\Delta \mathrm{EW}$ distributions for $\mathrm{CIV}_{\mathrm{S} 0}$ and $\mathrm{C}_{\mathrm{IV}} \mathrm{SA}$ troughs with similar EWs also differ from zero at more than $1 \sigma$. The means of the $\Delta \mathrm{EW}$ distributions for $\mathrm{CIV}_{\mathrm{S} 0}$ in the two different matching samples change from a positive value to a negative value. These results are initially surprising given the fact that the mean of the $\Delta \mathrm{EW}$ distribution for all $852 \mathrm{C}$ IV BAL troughs in our main sample is consistent with zero $(-0.0055 \pm 0.0373 \AA$; also see Section 4.4 of Filiz Ak et al. 2013). Indeed, the weakening and strengthening of BAL troughs are expected to be balanced to maintain an equilibrium population of BAL troughs in quasar spectra.

The differences between the mean values of the $\Delta \mathrm{EW}$ distributions can occur, for instance, if not only $|\Delta \mathrm{EW}|$ but also $\Delta \mathrm{EW}$ has a dependence on BAL-trough strength. To assess such dependency, we show $\Delta \mathrm{EW}$ as a function of first-epoch EW for $\mathrm{C}_{\mathrm{IV}_{00}}, \mathrm{C}_{\mathrm{IV}} \mathrm{SO}$, and $\mathrm{C}_{\text {IV }}$ SA troughs in Figure 13. The best fits of basic linearregression models are plotted in each panel of Figure 13 to demonstrate the apparent trends. Using Spearman rank-correlation tests (see Press et al. 2007), we find connections between $\Delta \mathrm{EW}$ and $\mathrm{EW}_{1}$ for $\mathrm{C}_{\mathrm{IV}_{00}}(P>99.9 \%)$ and likely also for $\mathrm{CIV}_{\mathrm{S} 0}(P=98.4 \%)$ troughs.

The apparent $\triangle \mathrm{EW}$ vs. $\mathrm{EW}_{1}$ relation primarily arises because the BAL-trough EW variation range is limited. Given that a BAL trough cannot weaken by more than its first-epoch EW and cannot strengthen by more than its second-epoch $\mathrm{EW}, \mathrm{EW}_{2}$, the variation strength is restricted to be $\mathrm{EW}_{2} \geq \Delta \mathrm{EW} \geq-\mathrm{EW}_{1}$. In addition, we exclude quasars that do not satisfy our necessary quasar- 
Table 8

Average values of BAL-trough properties for $\mathrm{C}_{\mathrm{IV}_{00}}, \mathrm{C}_{\mathrm{IV}} \mathrm{S}, \mathrm{C} \mathrm{IV}_{\mathrm{SA}}$, and all $\mathrm{C}$ IV BAL troughs

\begin{tabular}{ccccc}
\hline \hline & $\mathrm{C}_{\text {IV }}$ & $\mathrm{C}_{\text {IV }}$ & $\mathrm{C}_{\mathrm{S} 0}$ & All C IV \\
\hline$\langle\mathrm{EW}\rangle(\AA)$ & $4.76 \pm 0.25$ & $19.29 \pm 0.62$ & $32.38 \pm 1.35$ & $15.46 \pm 0.42$ \\
$\left\langle d_{\mathrm{BAL}}\right\rangle$ & $0.25 \pm 0.009$ & $0.47 \pm 0.009$ & $0.56 \pm 0.012$ & $0.41 \pm 0.006$ \\
$\Delta v\left(\mathrm{~km} \mathrm{~s}^{-1}\right)$ & $3940 \pm 182$ & $8600 \pm 255$ & $11668 \pm 428$ & $7195 \pm 146$ \\
\hline Number of Data Points & 113 & 246 & 95 & 852 \\
\hline
\end{tabular}

Uncertainties on the mean are calculated using the standard $\sigma / \sqrt{N}$ formula.

Table 9

Average values of BAL-trough velocities for $\mathrm{C}_{\mathrm{IV}_{00}}, \mathrm{C}_{\mathrm{IV}} \mathrm{S}_{\mathrm{S}}, \mathrm{C} \mathrm{IV}_{\mathrm{SA}}$, and all $\mathrm{C} \mathrm{IV}$ BAL troughs

\begin{tabular}{ccccc}
\hline \hline & $\mathrm{CIV}_{00}$ & $\mathrm{C}_{\mathrm{IV}}$ & $\mathrm{CIV}_{\mathrm{SA}}$ & All C IV \\
\hline$v_{\min }\left(\mathrm{km} \mathrm{s}^{-1}\right)$ & $-11898 \pm 400$ & $-7434 \pm 238$ & $-4838 \pm 241$ & $-8424 \pm 154$ \\
$v_{\max }\left(\mathrm{km} \mathrm{s}^{-1}\right)$ & $-15836 \pm 426$ & $-16035 \pm 308$ & $-16506 \pm 453$ & $-15619 \pm 168$ \\
$\left\langle v_{\text {cent }}\right\rangle\left(\mathrm{km} \mathrm{s}^{-1}\right)$ & $-13830 \pm 402$ & $-11998 \pm 235$ & $-11405 \pm 291$ & $-12229 \pm 140$ \\
\hline
\end{tabular}

\begin{tabular}{llcc} 
Number of Data Points & 113 & 246 & 95 \\
\hline Uncertainties on the mean are calculated using the standard $\sigma / \sqrt{N}$ formula. & Note that values \\
in this table are affected by censoring (see the text).
\end{tabular}

Table 10

Two-Sample Anderson-Darling Test Results for BAL-Trough Samples with Matching First-Epoch EWs ${ }^{\mathrm{a}}$

\begin{tabular}{cccrr}
\hline \hline & $\mathrm{C}_{\text {IV }} 00$ & C IV $_{\mathrm{S} 0}$ & C IV $_{\mathrm{S} 0}$ & $\mathrm{C}_{\text {IV }_{\mathrm{SA}}}$ \\
\hline Mean $\Delta \mathrm{EW}$ & $-1.13 \pm 0.47$ & $1.33 \pm 0.49$ & $-0.70 \pm 0.64$ & $0.75 \pm 0.38$ \\
Mean $|\Delta \mathrm{EW}|$ & $2.34 \pm 0.34$ & $1.84 \pm 0.45$ & $3.78 \pm 0.44$ & $2.11 \pm 0.29$ \\
$\sigma_{\Delta \mathrm{EW}}$ & $3.42 \pm 0.34$ & $3.14 \pm 0.35$ & $5.16 \pm 0.45$ & $3.09 \pm 0.27$ \\
$p_{\mathrm{AD}}$ & \multicolumn{2}{c}{$>9.9 \%$} & \multicolumn{2}{c}{$97.8 \%$} \\
Sample Size & \multicolumn{2}{c}{65} \\
\hline
\end{tabular}

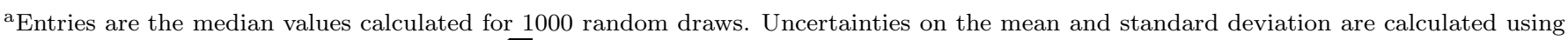
the standard $\sigma / \sqrt{N}$ formula and Equation 14.1.3 of Press et al. (2007), respectively. 


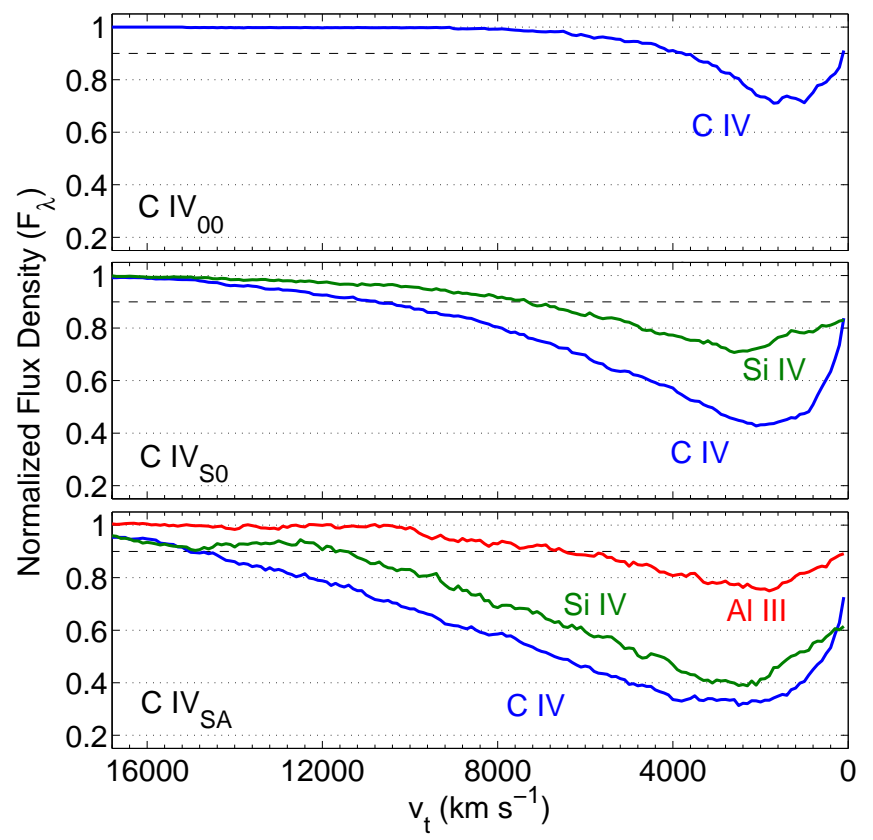

Figure 5. Composite BAL-trough profiles for $\mathrm{C}_{\mathrm{IV}} \mathrm{O0}$ (top), $\mathrm{C}_{\mathrm{IV}} \mathrm{SO}$ (middle), and CIV SA (bottom) BAL troughs. Solid curves show mean normalized flux density for CIV (blue), Si IV (green), and $\mathrm{Al}$ III (red) BAL troughs at overlapping velocities as a function of outflow velocity relative to $v_{\min }, v_{\mathrm{t}}=v-v_{\min }$. The horizontal dashed black lines in each panel show the level for $10 \%$ under the continuum, a level important for BAL-trough identification (See Equation 11). The composite profile shapes of $\mathrm{CIV}_{00}, \mathrm{C}_{\mathrm{IV}} \mathrm{SO}$, and C IVSA troughs differ significantly; stronger and wider C IV troughs are found when BAL troughs from lower ionization transitions are present.

selection criterion of $\mathrm{BI}_{3}^{20}>0$ in either epoch (see Section 2). Thus, this criterion leads to exclusion of disappearing (i.e., $\Delta \mathrm{EW} \approx-\mathrm{EW}_{1}$; e.g., Filiz Ak et al. 2012) and emerging (i.e., $\Delta \mathrm{EW} \approx \mathrm{EW}_{2}$ ) BAL troughs from our main sample.

In our matched samples, we must compare the $\Delta \mathrm{EW}$ distribution of the stronger $\mathrm{CIV}_{00}$ troughs with that of the weaker $\mathrm{CIV}_{\mathrm{S} 0}$ troughs, and that of the stronger $\mathrm{CIV}_{\mathrm{S} 0}$ troughs with that of the weaker $\mathrm{CIV}_{\mathrm{SA}}$ troughs. Therefore the differences in the means of these $\Delta \mathrm{EW}$ distributions are expected given the relations shown in Figure 13.

\subsection{EW Variation Correlations}

Previous studies (e.g., Gibson et al. 2010; Capellupo et al. 2012; Filiz Ak et al. 2013) have demonstrated that Si IV BAL troughs tend to vary in concert with C IV troughs at corresponding velocities and that the strength of fractional EW variations tends to be larger for Si IV BAL troughs. In order to investigate similar relations for $\mathrm{CIV}_{\mathrm{S} 0}$ (Section 4.6.1) and $\mathrm{CIV}_{\mathrm{SA}}$ (Section 4.6.2) troughs, we assess variation correlations between CIV troughs and corresponding Si IV and Al III troughs.

\subsubsection{EW Variation Correlations for the $\mathrm{CIV}_{\mathrm{S} 0}$ Sample}

Figure 14 presents the $\Delta \mathrm{EW}$ and $\Delta \mathrm{EW} /\langle\mathrm{EW}\rangle$ correlations for $\mathrm{CIV}_{\mathrm{S} 0}$ and the corresponding $\mathrm{Si} I V$ troughs. Consistent with the findings of Filiz Ak et al. (2013) for all CIV troughs, the Spearman-test results show that both the $\Delta \mathrm{EW}$ and $\Delta \mathrm{EW} /\langle\mathrm{EW}\rangle$ correlations are highly significant $(P>99.9 \%)$. Given the apparent scatter in both panels of Figure14 we determine the best fits using a Bayesian linear-regression model that considers the intrinsic scatter of the sample (Kelly 2007). We found the following relations, calculating the mean and the standard deviation of the linear-regression model parameters for 10,000 random draws from the sample:

$$
\begin{aligned}
& \Delta \mathrm{EW}_{\mathrm{CIV}_{\mathrm{So}}}=(1.20 \pm 0.082) \times \Delta \mathrm{EW}_{\mathrm{Si} \mathrm{IV}} \\
&+(0.051 \pm 0.216), \quad \sigma_{\mathrm{IS}}=3.4 \AA \\
& \frac{\Delta \mathrm{EW}}{\langle\mathrm{EW}\rangle_{\mathrm{C} \mathrm{IV}_{\mathrm{SO}}}}=(0.515 \pm 0.023) \times \frac{\Delta \mathrm{EW}}{\langle\mathrm{EW}\rangle_{\mathrm{Si} \mathrm{IV}}} \\
&+(0.006 \pm 0.011), \quad \sigma_{\mathrm{IS}}=0.15 .
\end{aligned}
$$

Here $\sigma_{\text {IS }}$ is the standard deviation of the intrinsic scatter. The slopes of the $\Delta \mathrm{EW}$ and $\Delta \mathrm{EW} /\langle\mathrm{EW}\rangle$ correlations are consistent with the findings in Section 4.6 of Filiz Ak et al. (2013) within $1 \sigma$, indicating that the EW variations of $\mathrm{CIV}_{\mathrm{S} 0}$ troughs are comparable to those of Si IV troughs, whereas the fractional EW variations of $\mathrm{CIV}_{\text {So }}$ troughs are approximately half of those of Si IV troughs. The EW variations for $\mathrm{CIV}_{\mathrm{S} 0}$ vs. corresponding Si IV troughs are not highly inconsistent with being equal; the deviation between the best fit and equality is $\approx 2.4 \sigma$ and the intrinsic scatter is large.

\subsubsection{EW Variation Correlations for the $\mathrm{CIV}_{\mathrm{SA}}$ Sample}

Figure 15 displays the $\Delta \mathrm{EW}$ and $\Delta \mathrm{EW} /\langle\mathrm{EW}\rangle$ relations for $\mathrm{CIV}_{\mathrm{SA}} \mathrm{BAL}$ troughs and the corresponding Si IV and Al III BAL troughs. The Spearman-test results indicate highly significant $(P>99.9 \%)$ correlations for both $\Delta \mathrm{EW}$ and $\Delta \mathrm{EW} /\langle\mathrm{EW}\rangle$ between $\mathrm{CIV}_{\mathrm{SA}}$ and the corresponding Si IV troughs. The $\Delta \mathrm{EW}$ correlation between $\mathrm{C}_{\mathrm{IV}_{\mathrm{SA}}}$ and the corresponding $\mathrm{Al}$ III troughs is marginally significant $(P=99.8 \%)$, while the $\Delta \mathrm{EW} /\langle\mathrm{EW}\rangle$ correlation is highly significant $(P>99.9 \%)$. The $\Delta \mathrm{EW}$ correlation between $\mathrm{CIV}_{\mathrm{SA}}$ and $\mathrm{Al}$ III troughs is not highly significant, possibly because of the small range of $\Delta \mathrm{EW}$ values for both ions. In addition to this effect, the relatively small number of data points in the C IV SA sample, and the apparently large intrinsic scatter of the $\Delta \mathrm{EW}$ values, may hide any possible correlation. The following relations are found using the Bayesian linear-regression fit:

$$
\begin{aligned}
\Delta \mathrm{EW}_{\mathrm{CIV}_{\mathrm{SA}}} & =(0.646 \pm 0.081) \times \Delta \mathrm{EW}_{\mathrm{Si} \text { IV }} \\
+ & (0.080 \pm 0.280), \quad \sigma_{\mathrm{IS}}=2.5 \AA \\
\frac{\Delta \mathrm{EW}}{\langle\mathrm{EW}\rangle_{\mathrm{CIV}_{\mathrm{SA}}}} & =(0.355 \pm 0.044) \times \frac{\Delta \mathrm{EW}}{\langle\mathrm{EW}\rangle_{\mathrm{Si} \mathrm{IV}}} \\
& -(0.002 \pm 0.008), \quad \sigma_{\mathrm{IS}}=0.08
\end{aligned}
$$

$$
\begin{aligned}
\Delta \mathrm{EW}_{\mathrm{CIV}_{\mathrm{SA}}} & =(0.172 \pm 0.171) \times \Delta \mathrm{EW}_{\mathrm{Al} \text { III }} \\
+ & (0.493 \pm 0.348), \quad \sigma_{\mathrm{IS}}=3.3 \AA \\
\frac{\Delta \mathrm{EW}}{\langle\mathrm{EW}} \overline{\mathrm{CIV}}_{\mathrm{SA}} & =(0.111 \pm 0.035) \times \frac{\Delta \mathrm{EW}}{\langle\mathrm{EW}} \mathrm{Al} \mathrm{III}
\end{aligned}
$$



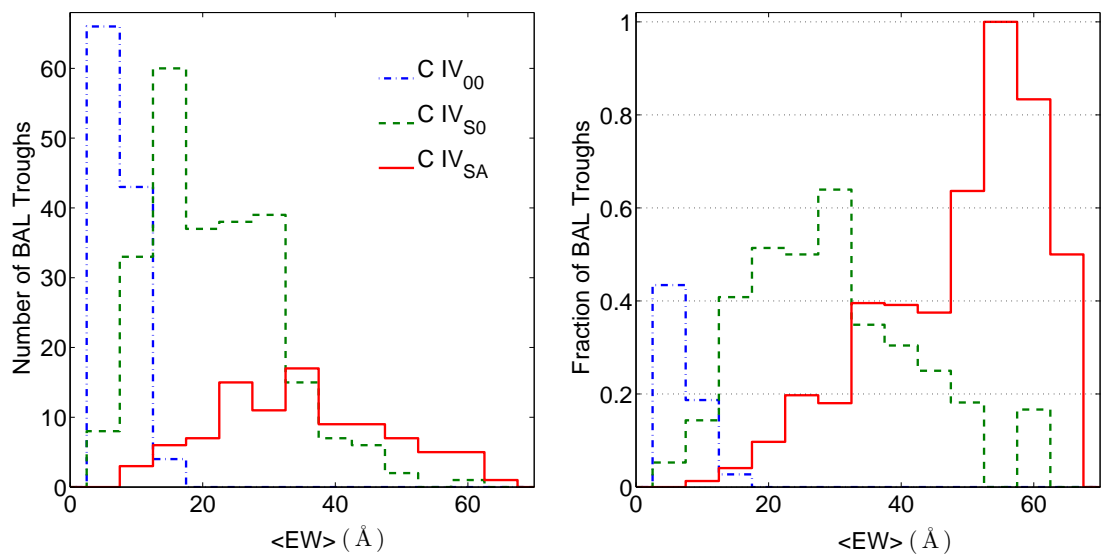

Figure 6. Average EW, $\langle\mathrm{EW}\rangle$, distributions for $\mathrm{CIV}_{00}$ (dot-dashed blue), $\mathrm{C}_{\mathrm{IV}}$ (dashed green), and $\mathrm{C}_{\mathrm{S}}$ SA (solid red) BAL troughs. The right panel shows the fraction of ${\mathrm{C} I V_{00}}, \mathrm{C}_{\mathrm{IV}} \mathrm{S}$, and $\mathrm{C}_{\mathrm{IV}} \mathrm{SA}$ BAL troughs to all $852 \mathrm{C}$ IV BAL troughs in our sample. Since troughs with mini-BALs are excluded, the numbers in the right panel need not sum to unity for a given bin. The $\langle\mathrm{EW}\rangle$ distributions of the three C IV groups are significantly (99.9\%) different from each other.
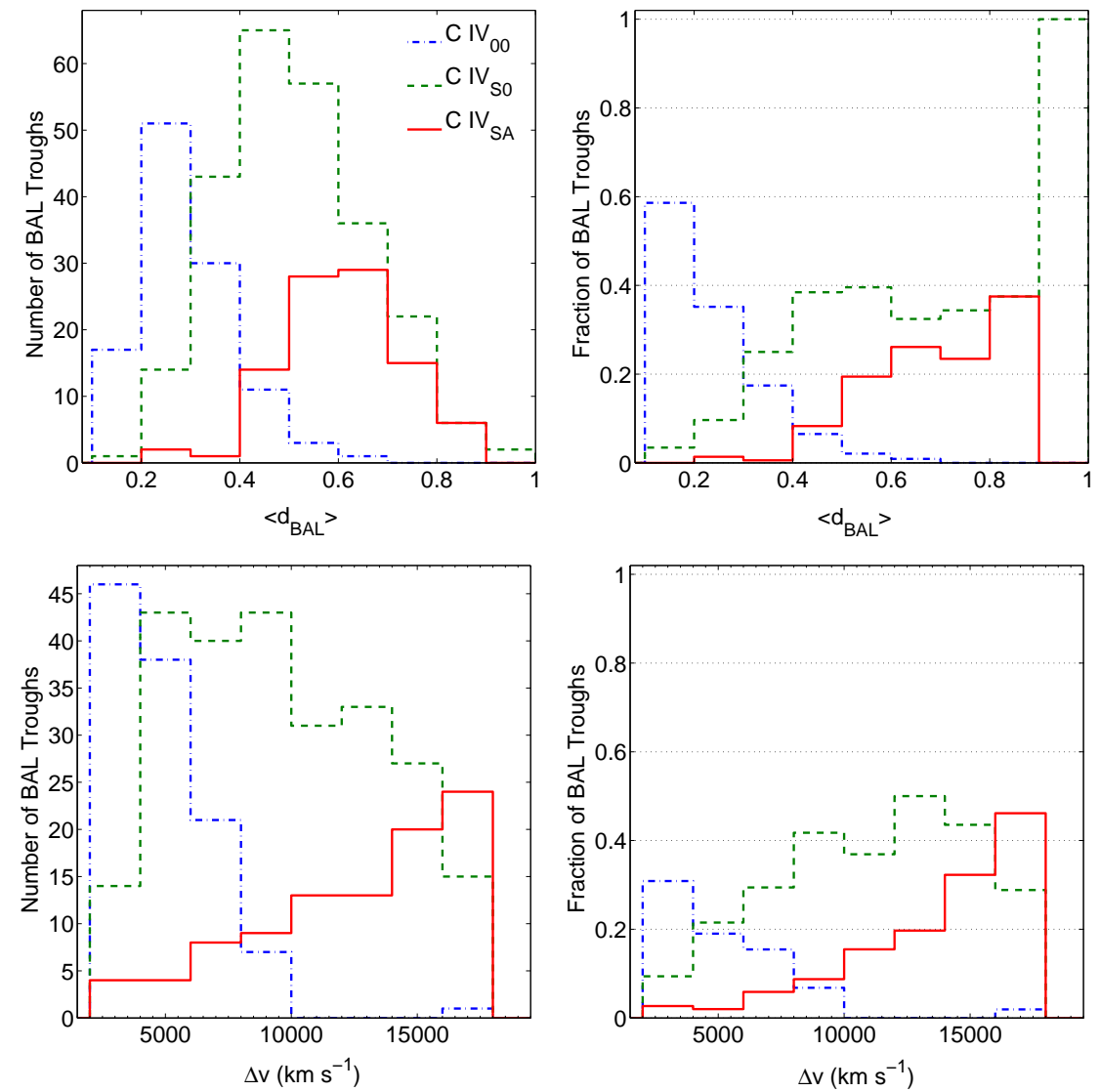

Figure 7. Average depth, $\left\langle d_{\mathrm{BAL}}\right\rangle$ (upper panels), and velocity width, $\Delta v$ (lower panels), distributions for $\mathrm{C}_{\mathrm{IV}_{00}}$ (dot-dashed blue), $\mathrm{C}_{\mathrm{IV}} \mathrm{S} 0$ (dashed green), and $\mathrm{C}_{\mathrm{IV}} \mathrm{SA}$ (solid red) BAL troughs. The right panels show the fraction of $\mathrm{C}_{\mathrm{IV}} \mathrm{V}_{00}, \mathrm{C}_{\mathrm{IV}} \mathrm{S}$, and $\mathrm{C}_{\mathrm{IV}} \mathrm{SA} \mathrm{BAL}$ troughs relative to all $\mathrm{C}_{\mathrm{IV}} \mathrm{BAL}$ troughs in our main sample. The $\left\langle d_{\mathrm{BAL}}\right\rangle$ and $\Delta v$ distributions for $\mathrm{C}_{\mathrm{IV}} 00, \mathrm{C}_{\mathrm{IV}} \mathrm{S}_{0}$, and $\mathrm{C}_{\mathrm{IV}} \mathrm{SA}_{\mathrm{BAL}}$ troughs are significantly $(99.9 \%)$ different from each other.

$$
+(0.005 \pm 0.011), \quad \sigma_{\text {IS }}=0.10 .
$$

Figure 15 presents the best fits of the CIV and Si IV variation relations both for the $\mathrm{CIV}_{\mathrm{SO}}$ and the $\mathrm{CIV}_{\mathrm{SA}}$ samples for comparison purposes. Consistent with the results of our investigations in Section 4.5 , the ranges of $\Delta \mathrm{EW}_{\mathrm{C} \text { IV }}$ and $\Delta \mathrm{EW} /\langle\mathrm{EW}\rangle_{\mathrm{C} \text { IV }}$ in Figures 14 and 15] indicate that $\mathrm{CIV}_{\mathrm{SA}}$ troughs tend to be less variable than $\mathrm{C}_{\mathrm{IV}_{\mathrm{S}} 0}$ troughs. Moreover, the ranges of $\Delta \mathrm{EW}_{\mathrm{Si} I V}$ and
$\Delta \mathrm{EW} /\langle\mathrm{EW}\rangle_{\mathrm{SiIV}}$ in Figures 14 and 15 suggest that the Si IV BAL troughs of the $\mathrm{C}_{\mathrm{IV}} \mathrm{SA}$ sample show less variation than those of the $\mathrm{C}_{\mathrm{IV}} \mathrm{So}$ sample. The flat slopes of Equations 4 and 6 indicate that the $\mathrm{CIV}_{\mathrm{SA}}$ troughs tend to show very small variations. The slopes of Equations 5 and 7 indicate that $\mathrm{Al}$ III troughs tend to be more fractionally variable than both the corresponding $\mathrm{C} \mathrm{IV}_{\mathrm{SA}}$ and Si IV troughs, and Si IV troughs tend to be more fraction- 

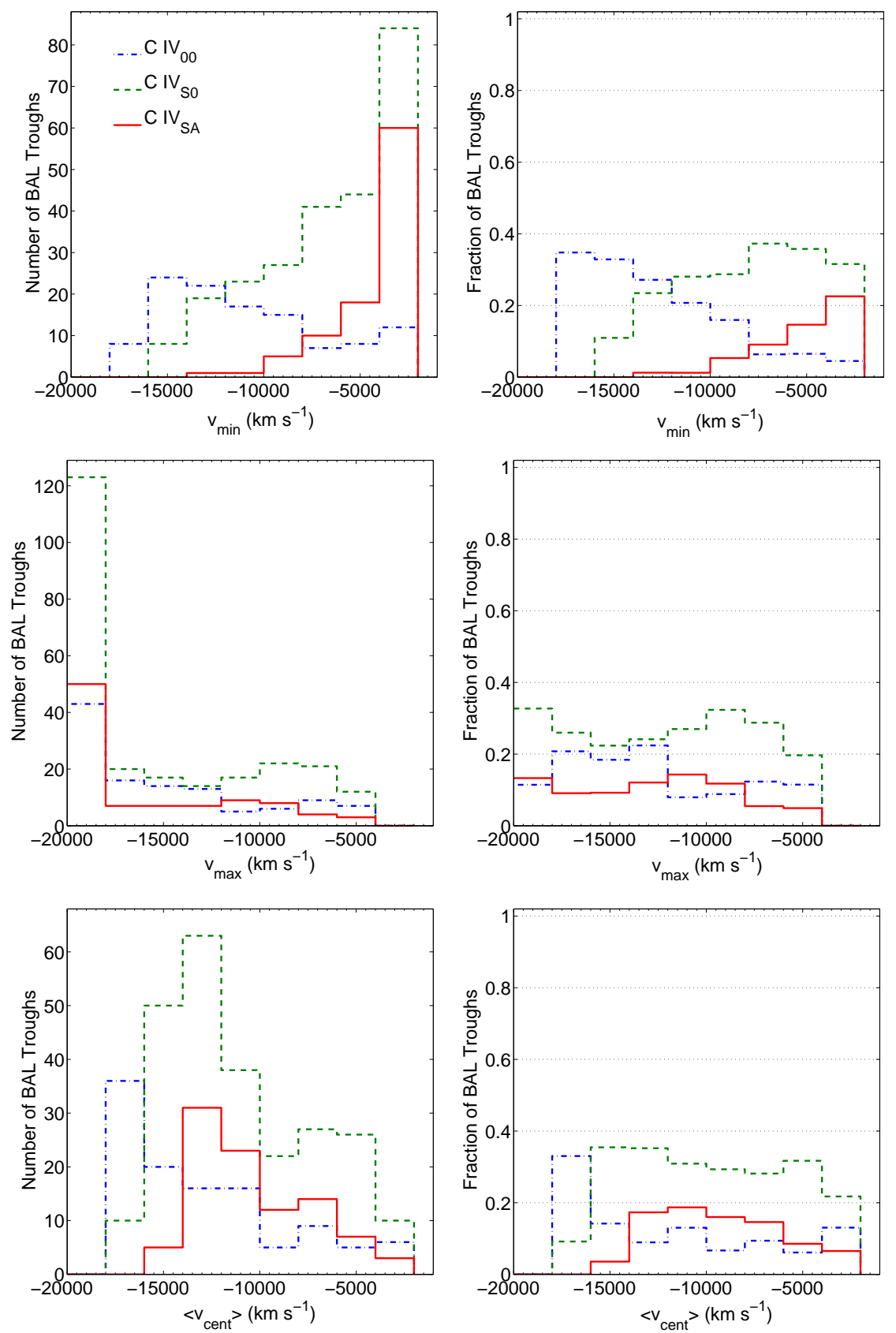

Figure 8. Minimum velocity, $v_{\min }$ (upper panels), maximum velocity, $v_{\max }$ (middle panels), and average centroid velocity, $\left\langle v_{\text {cent }}\right\rangle($ (lower panels), distributions for $\mathrm{C}_{\mathrm{V}} 00$ (dot-dashed blue), $\mathrm{C}_{\mathrm{IV}}$ (dashed green), and C IVSA (solid red) BAL troughs. The right panels display the fraction of $\mathrm{C}_{\mathrm{IV}} \mathrm{V}_{0}, \mathrm{C}_{\mathrm{IV}} \mathrm{SO}$, and $\mathrm{C}_{\mathrm{IV} \mathrm{SA}} \mathrm{BAL}$ troughs relative to all C IV BAL troughs in our main sample. The $v_{\min }$ and $\left\langle v_{\text {cent }}\right\rangle$ distributions for $\mathrm{CIV}_{00}, \mathrm{CIV}_{\mathrm{S} 0}$, and $\mathrm{CIV}_{\mathrm{SA}}$ BAL troughs are significantly $(99.9 \%)$ different from each other, whereas the $v_{\text {max }}$ distributions do not show highly significant differences. 

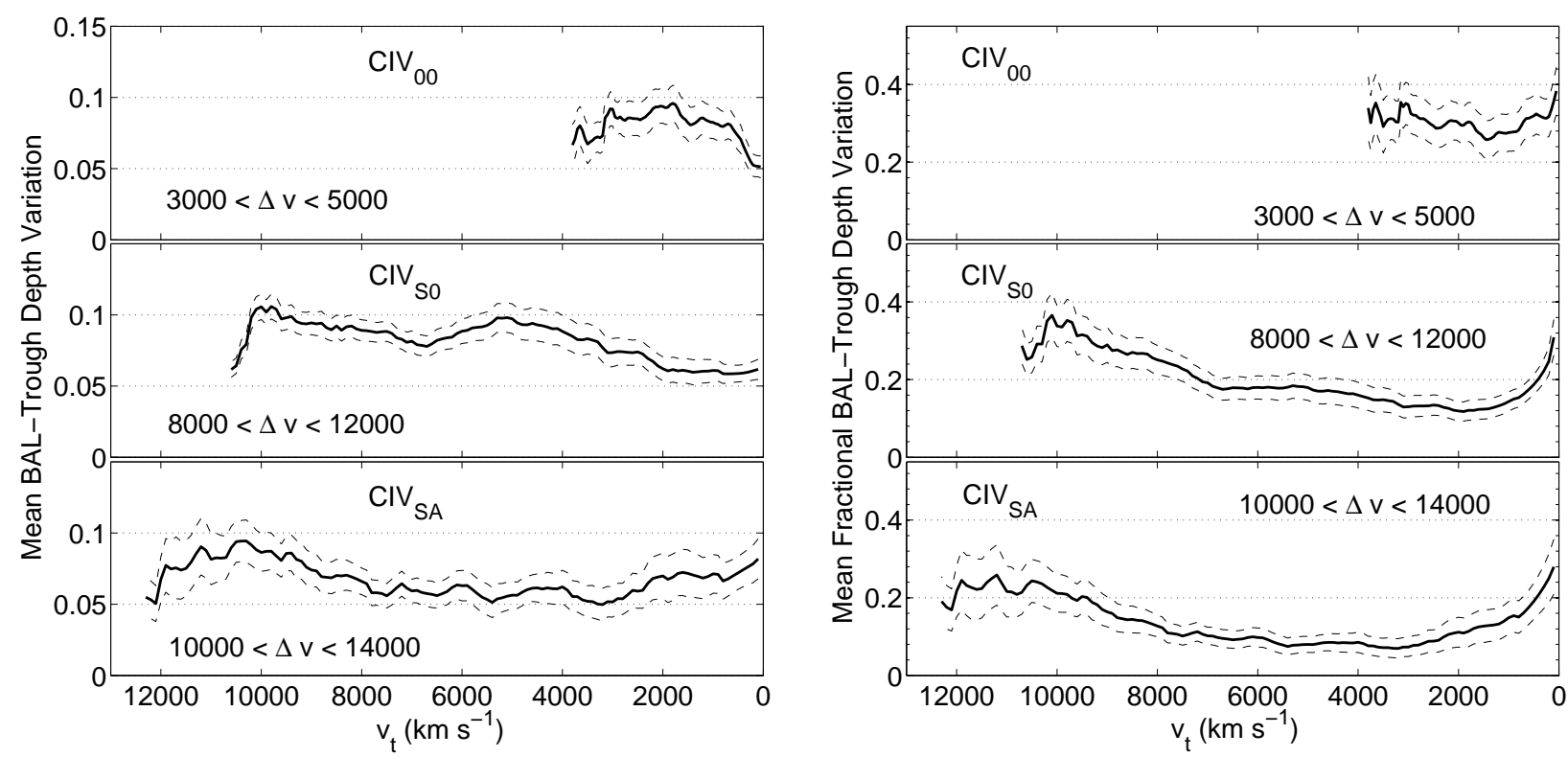

Figure 9. BAL-trough variation profiles for $\mathrm{C}_{\text {IV }} 00$ troughs with $3000<\Delta v<5000$ (top panels), $\mathrm{C}_{\text {IV }}$ So troughs with $8000<\Delta v<12000$ (middle panels), and $\mathrm{C}_{\mathrm{IV}} \mathrm{SA}$ troughs with $10000<\Delta v<14000$ (bottom panels) in $\mathrm{km} \mathrm{s}^{-1}$. Left panels: Solid-black curves show the mean composite profiles for absolute depth variations of $\mathrm{C}$ IV BAL troughs as a function of outflow velocity, $v_{\mathrm{t}}$. The dashed curves show the error on the mean. Right panels: Mean composite profiles for absolute fractional depth variations of CIV BAL troughs, where the fractional variation is the depth variation divided by the average depth. While the $\mathrm{C}_{\mathrm{IV}} \mathrm{00}$ troughs show similar variability across the entire trough, the $\mathrm{CIV}_{\mathrm{S} 0}$ and $\mathrm{C} \mathrm{IV}_{\mathrm{SA}}$ troughs show less variability at lower velocities where the $\mathrm{Si}$ IV and $\mathrm{Al}$ III absorption tend to be the strongest.

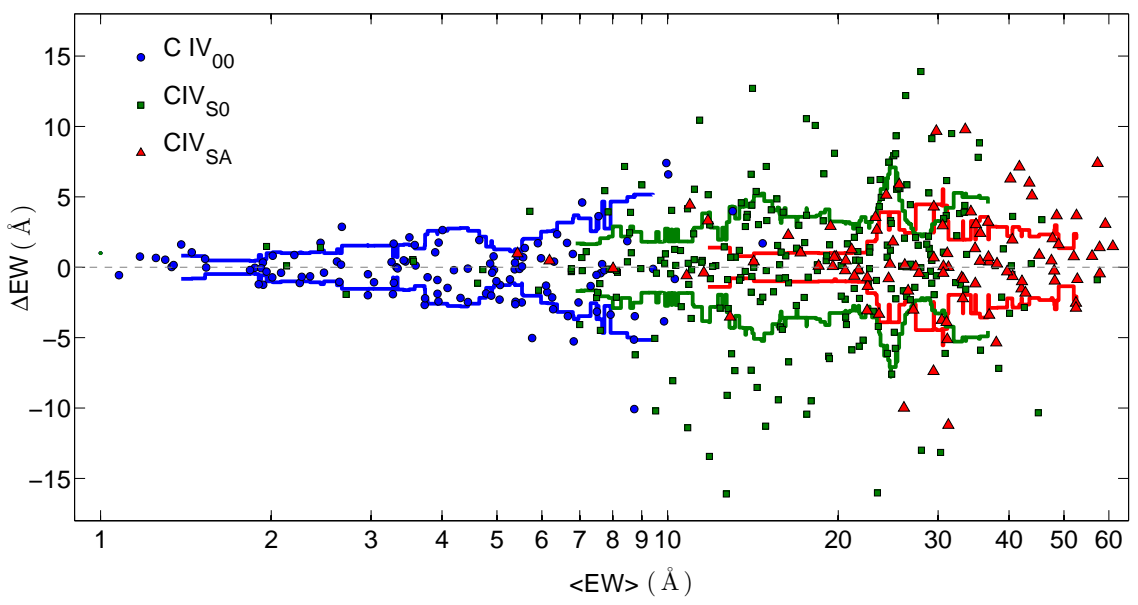

Figure 10. $\Delta \mathrm{EW}$ vs. $\langle\mathrm{EW}\rangle$ for $\mathrm{CIV}_{00}$ (blue circles), $\mathrm{C}_{\mathrm{IV}}$ (green squares), and $\mathrm{C}_{\mathrm{IV}} \mathrm{SA}$ (red triangles) BAL troughs. Solid curves show rms values calculated with a sliding window containing $10 \%$ of the total data points. The spread of $\Delta \mathrm{EW}$ generally increases with increasing $\langle\mathrm{EW}\rangle$ for $\mathrm{CIV}_{00}, \mathrm{CIV}_{\mathrm{S} 0}$, and $\mathrm{CIV}_{\mathrm{SA}}$ troughs. In overlapping $\langle\mathrm{EW}\rangle$ ranges, $\mathrm{CIV}_{00}$ troughs tend to be more variable than $\mathrm{C}_{\mathrm{IV}} \mathrm{So}$ troughs, and similarly $\mathrm{C}_{\mathrm{IV}} \mathrm{So}$ troughs tend to be more variable than $\mathrm{C}_{\mathrm{IV}} \mathrm{SA}$ troughs.

ally variable than the corresponding $\mathrm{C}_{\mathrm{IV}_{\mathrm{SA}}}$ troughs.

\section{SUMMARY OF RESULTS, DISCUSSION, AND FUTURE WORK}

We have investigated the profiles, standard characteristic properties, and variation behaviors of $\mathrm{C}$ IV BAL troughs, considering how these change when BAL troughs from Si IV and Al III are present at corresponding velocities. We have utilized a sample of $852 \mathrm{C} \mathrm{IV}$ BAL troughs; 113 of these have no detection of any corresponding SiIV or AlIII BALs or mini-BALs in both epochs ( $\mathrm{CIV}_{00}$ troughs), 246 of these are accompanied by a Si IV BAL trough but have no corresponding Al III BALs or mini-BALs ( IV $_{\text {So }}$ troughs), and 95 of these are accompanied by both SiIV and AliII BALs (C IVSA troughs). The main observational findings of our study are the following:

1. The composite profiles of $\mathrm{C}_{\mathrm{IV}_{00}}, \mathrm{C}_{\mathrm{IV}_{\mathrm{S}}}$, and $\mathrm{C}_{\mathrm{IV}} \mathrm{SA}$ troughs differ significantly; stronger C IV troughs are found when accompanying BAL troughs from lower ionization transitions are present. Furthermore, the composite profiles for $\mathrm{CIV}_{\mathrm{S} 0}$ and $\mathrm{CIV}_{\mathrm{SA}}$ troughs are deeper at the lower velocities where $\mathrm{Al}$ III and, to a lesser extent, Si IV troughs are preferentially found. See Section 4.1 .

2. The two-epoch average $\mathrm{EW},\langle\mathrm{EW}\rangle$, distributions for $\mathrm{CIV}_{00}, \mathrm{CIV}_{\mathrm{S} 0}$, and $\mathrm{CIV}_{\mathrm{SA}} \mathrm{BAL}$ troughs are significantly $(>99.9 \%)$ different. Generally, $\mathrm{C}_{\mathrm{IV}_{00}}$ 


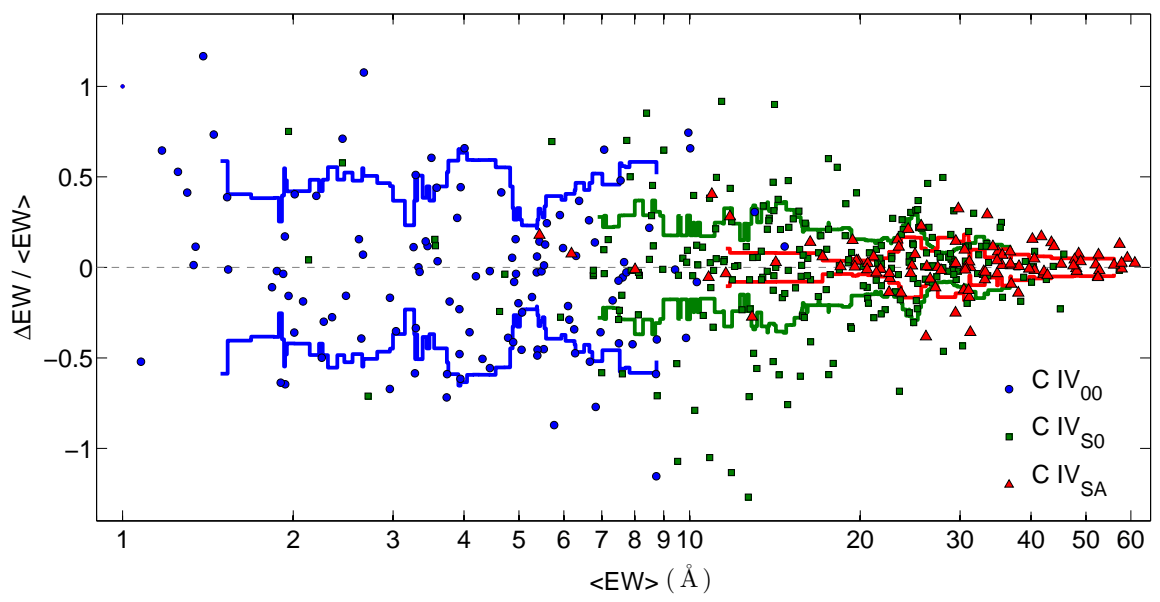

Figure 11. Same as Figure 10 but for $\Delta \mathrm{EW} /\langle\mathrm{EW}\rangle$. The spread of $\Delta \mathrm{EW} /\langle\mathrm{EW}\rangle$ decreases with increasing $\langle\mathrm{EW}\rangle$. In overlapping $\langle\mathrm{EW}\rangle$ ranges, $\mathrm{C}_{\mathrm{V}} 00$ troughs tend to be fractionally more variable than $\mathrm{CIV}_{\mathrm{S} 0}$ troughs, and similarly $\mathrm{C}_{\mathrm{V}} \mathrm{So}$ troughs tend to be fractionally more variable than CIVSA troughs.
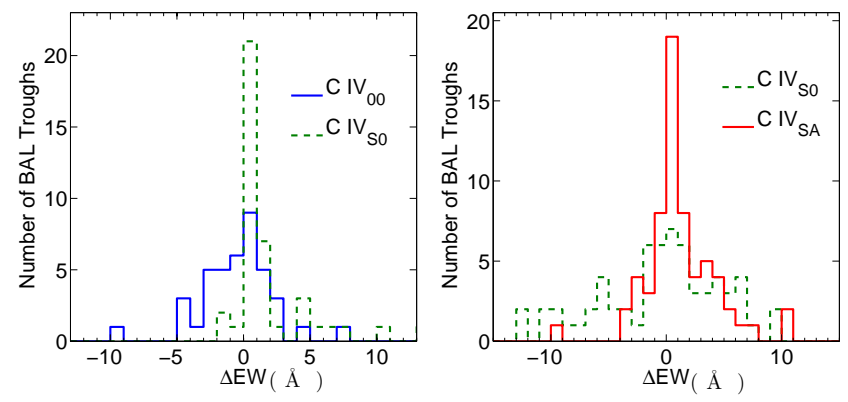

Figure 12. $\triangle \mathrm{EW}$ distributions for $\mathrm{C}_{\mathrm{IV}} 00$ vs. $\mathrm{C}_{\mathrm{IV}} \mathrm{S}$ troughs (left panel) and for $\mathrm{CIV}_{\mathrm{S} 0}$ vs. CIV $\mathrm{SA}$ troughs (right panel), where the samples show BAL troughs with similar EWs. CIV00 troughs are more variable than $\mathrm{C}_{\mathrm{IV}_{\mathrm{S}}}$ troughs, while $\mathrm{C}_{\mathrm{IV}} \mathrm{So}$ troughs are in turn more variable than $\mathrm{C}_{\mathrm{IV}} \mathrm{SA}$ troughs.

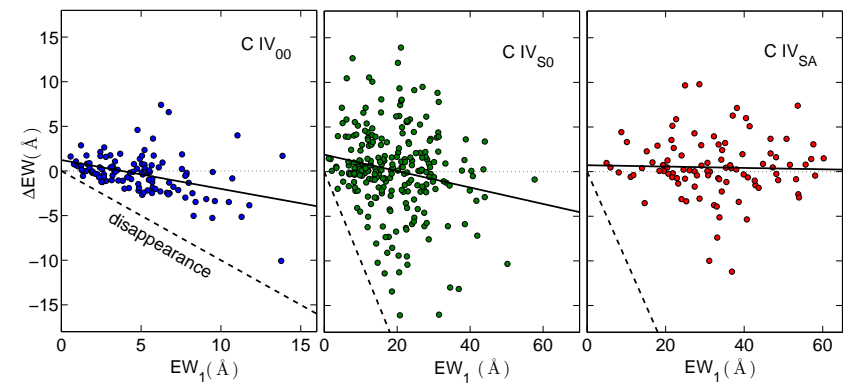

Figure 13. $\triangle \mathrm{EW}$ as a function of $\mathrm{EW}_{1}$ for $\mathrm{C}_{\mathrm{IV}} 00$ (left), $\mathrm{C}_{\mathrm{IV}} \mathrm{S}$ (middle), and $\mathrm{CIV}_{\mathrm{SA}}$ (right) BAL troughs. The solid lines show the best fits of basic linear-regression models in each panel. The dashed lines denote where $\Delta \mathrm{EW}=-\mathrm{EW}_{1}$ (corresponding to $\mathrm{BAL}$ disappearance). The apparent connection between $\Delta \mathrm{EW}$ and $\mathrm{EW}_{1}$ arises primarily because a BAL trough cannot weaken by more than its first-epoch EW.

troughs have small EWs, C IV $\mathrm{S}_{0}$ troughs have moderate EWs, and $\mathrm{CIV}_{\mathrm{SA}}$ troughs have large EWs. We find that increases in both depth and velocity width contribute comparably to the increase in EW from $\mathrm{CIV}_{00}$ to $\mathrm{CIV}_{\mathrm{S} 0}$ to $\mathrm{CIV}_{\mathrm{SA}}$ troughs. See Section 4.2 .

3 . The minimum and average centroid velocities decrease from $\mathrm{C}_{\mathrm{IV}} 00$ to $\mathrm{C}_{\mathrm{IV}} \mathrm{S}$ to $\mathrm{C}_{\mathrm{IV}} \mathrm{SA}$ troughs; this decrease is most notable for the minimum velocity, which changes on average by a factor of $\approx 2.5$, while the decrease is mild $(\approx 25 \%)$ for the average centroid velocity. See Section 4.3 .

4. Composite depth-variation and fractional-depthvariation profiles have been used to investigate the relative variability of $\mathrm{C}_{\mathrm{IV}_{00}}, \mathrm{CIV}_{\mathrm{S} 0}$, and $\mathrm{CIV}_{\mathrm{SA}}$ $\mathrm{BAL}$ troughs. BAL variability generally decreases from $\mathrm{CIV}_{00}$ to $\mathrm{CIV}_{\mathrm{S} 0}$ to $\mathrm{CIV}_{\mathrm{SA}}$ BALs, particularly in a fractional sense. For $\mathrm{CIV}_{\mathrm{S} 0}$ and $\mathrm{CIV}_{\mathrm{SA}}$ troughs, the lower velocity portions of the troughs tend to be the least variable, and these are the regions where $\mathrm{Al}$ III and, to a lesser extent, Si IV troughs are preferentially found. See Section 4.4.

5 . The spread of $\Delta \mathrm{EW}$ generally increases with increasing $\langle\mathrm{EW}\rangle$, and the spread of $\Delta \mathrm{EW} /\langle\mathrm{EW}\rangle$ generally decreases with increasing $\langle\mathrm{EW}\rangle$, for $\mathrm{CIV}_{00}$, $\mathrm{C}_{\mathrm{IV}}$, and $\mathrm{CIV}_{\mathrm{SA}}$ BAL troughs; this result is consistent with the general behavior of all $\mathrm{C}$ IV $\mathrm{BAL}$ troughs (e.g., Filiz Ak et al. 2013). In overlapping ranges of $\langle\mathrm{EW}\rangle, \mathrm{C}_{\mathrm{IV}} 00$ troughs appear to vary more strongly than $\mathrm{CIV}_{\mathrm{S} 0}$ troughs $(P>99.9 \%)$, and similarly $\mathrm{CIV}_{\mathrm{S} 0}$ troughs appear to vary more strongly than $\mathrm{C}_{\mathrm{IV}}$ A troughs $(P=97.8 \%)$. See Section 4.5.1.

6. For a proper comparison of the variation characteristics of the three CIV groups, we compare $\triangle \mathrm{EW}$ distributions of samples of $\mathrm{BAL}$ troughs with matched first-epoch EWs. $\mathrm{CIV}_{\mathrm{S} 0}$ troughs are somewhat less variable than $\mathrm{C}_{\mathrm{IV}} 00$ troughs with matched EWs, and CIVSA troughs are substantially less variable than $\mathrm{CIV}_{\mathrm{S} 0}$ troughs with matched EWs. See Section 4.5.2.

7. The Si IV BAL troughs associated with the $\mathrm{CIV}_{\mathrm{SO}}$ sample show EW and fractional EW variations that are generally in concert with those of the corresponding $\mathrm{C}_{\mathrm{IV}} \mathrm{So}$ troughs. We quantify the relevant correlations and find that Si IV troughs show similar EW and larger fractional EW variations than corresponding $\mathrm{C}_{\mathrm{IV}} \mathrm{S}$ troughs. See Section 4.6.1,

8. The EW and fractional $\mathrm{EW}$ variations of $\mathrm{Al} \mathrm{III}$ troughs are less clearly linked with those of $\mathrm{C}_{\mathrm{IV}} \mathrm{SA}$ 

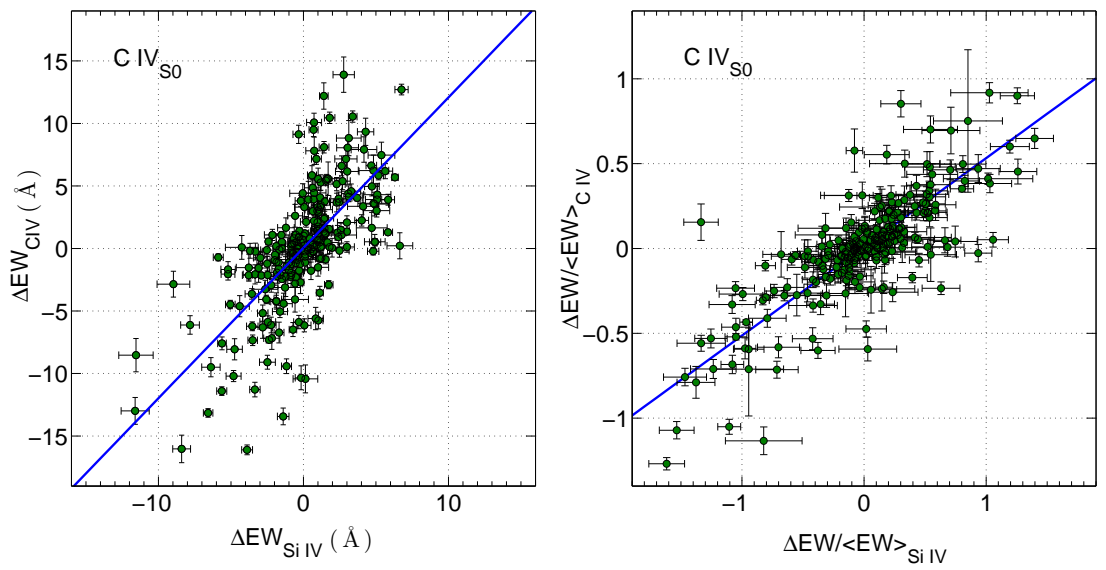

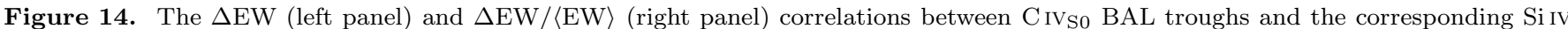
BAL troughs. Spearman-test results show highly significant correlations $(P>99.9 \%)$ for both panels. The solid-blue lines show the best fit found using a Bayesian linear-regression model.
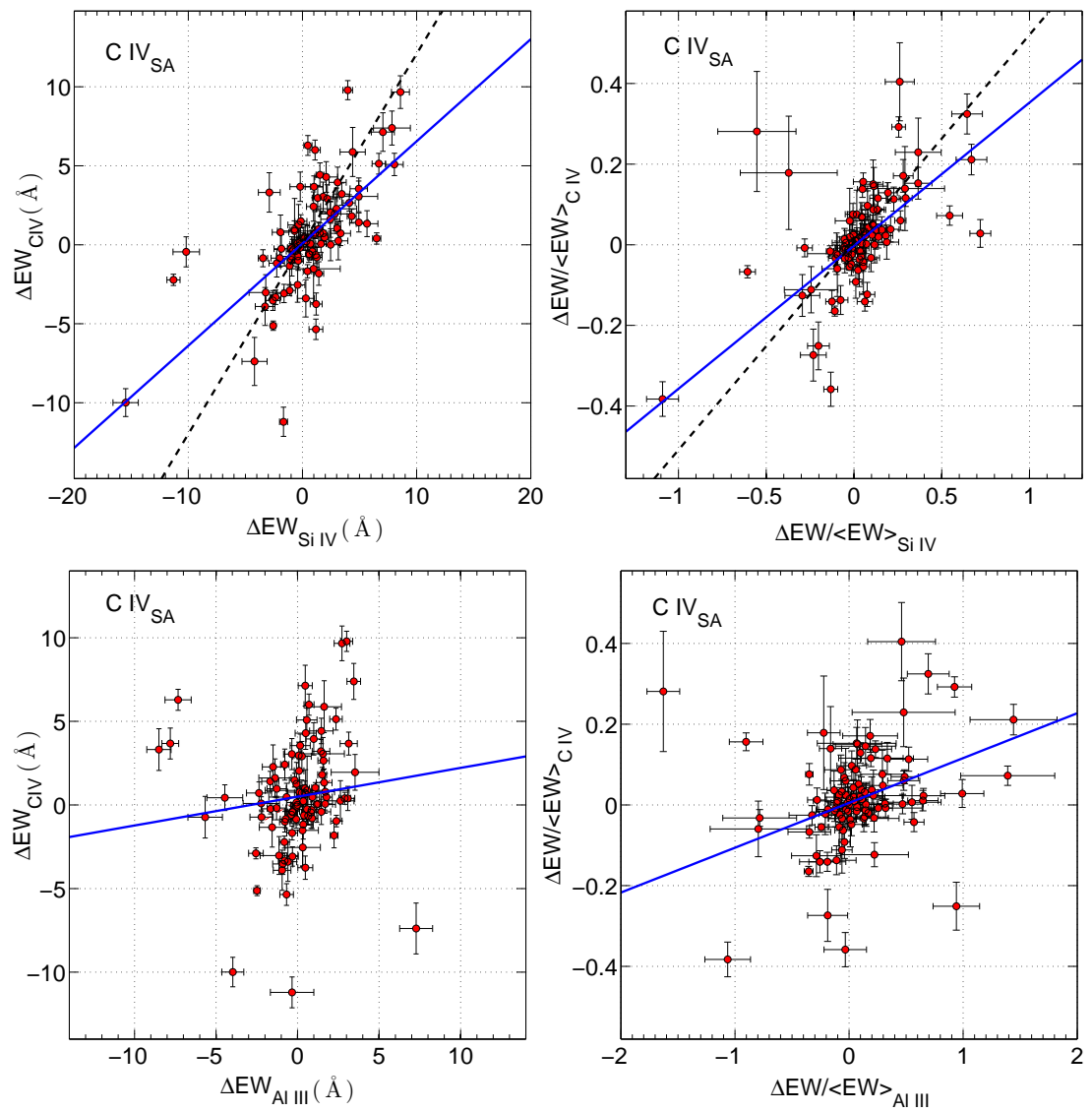

Figure 15. The $\Delta \mathrm{EW}$ (left panels) and $\Delta \mathrm{EW} /\langle\mathrm{EW}\rangle$ (right panels) correlations for $\mathrm{C}_{\mathrm{IV}} \mathrm{SA}$ vs. Si IV troughs (top panels) and $\mathrm{C}$ IV $\mathrm{SA}$ vs. Al III troughs (bottom panels). Spearman-test results show highly significant (both with $P>99.9 \%$ ) $\Delta \mathrm{EW}$ and $\Delta \mathrm{EW} /\langle\mathrm{EW}\rangle$ correlations for $\mathrm{C}_{\text {IV }}$ S vs. Si IV troughs, and a significant $\Delta \mathrm{EW} /\langle\mathrm{EW}\rangle$ correlation for $\mathrm{C}_{\text {IVSA }}$ vs. Al III troughs, whereas the $\Delta \mathrm{EW}$ correlation for $\mathrm{C}$ IVSA vs. Al III troughs is marginally significant with $P=99.8 \%$. The solid-blue lines show the best-fit relations in each panel. The dashed-black lines in the top two panels show the best-fit models for $\mathrm{C}_{\mathrm{IV}} \mathrm{SO}$ BAL troughs (Equations 2 and 3 ) for comparison.

troughs, although correlation testing does indicate some correspondence. Al III troughs show larger EW and fractional EW variations than corresponding $\mathrm{CIV}_{\mathrm{SA}}$ and Si IV troughs. See Section 4.6.2.

We now examine the implications of our observational findings considering the best-developed model for quasar BAL outflows, that of an equatorial radiation-driven disk wind (e.g., Murrav et al. 1995; Proga et al. 2000;
Higginbottom et al. 2013, see also Section 11). While other scenarios for BAL outflows also exist, such as those proposing that BALs primarily are formed at large distances $(0.1-10 \mathrm{kpc}$ ) from the SMBH (e.g., Arav et al. 2013; also see Faucher-Giguère et al. 2012; but see Section 5.3 of Lucy et al. 2014 for a critique), these have not been developed via numerical simulations to the point where robust comparisons with our observational find- 
ings are possible. Of course, any model for BAL winds, current or future, can be usefully constrained by our observational results presented above.

Figure 16] shows density and poloidal velocity maps of the disk-wind model. In this figure, two lines-of-sight are marked, corresponding to different viewing inclinations, along which an observer would see $\mathrm{CIV}_{00}$ and $\mathrm{CIV}_{\mathrm{SA}}$ troughs; in parallel with our subscript notation for C IV troughs, we will refer to these lines-of-sight (LOS) as $\mathrm{LOS}_{00}$ and $\mathrm{LOS}_{\mathrm{SA}}$, respectively, throughout. We expect generally correlated changes of ionization level, kinematics, and column density as our line-of-sight is varied from $\mathrm{LOS}_{00}$ to $\mathrm{LOS}_{\mathrm{SA}}$ (see Section 1), and this is relevant to our discussion below 18 We recognize that there is strong observational evidence supporting intrinsic object-to-object differences as well as inclination effects among BAL quasars (e.g., Boroson \& Mevers 1992; Turnshek et al. 1994; Zhang et al. 2010; DiPompeo et al. 2012). For example, the probability of observing a $\mathrm{C}_{\text {IV }}$ A trough will be higher for objects having a larger global covering factor of low-ionization gas (cf., the weak [O III] objects discussed in Boroson \& Mevers 1992) 19 However, such global-covering-factor effects do not affect our main reasoning below which is focused upon the typical measured properties of $\mathrm{CIV}_{00}$ vs. CIV 0 vs. C IV troughs rather than how often each of these trough types is observed.

Considering the $\mathrm{LOS}_{00}$ and $\mathrm{LOS}_{\mathrm{SA}}$ lines-of-sight in Figure 16 we present below a comparative assessment of the expected properties of $\mathrm{CIV}_{00}$ and $\mathrm{CIV}_{\mathrm{SA}}$ troughs, relating these to the observational findings above (we then discuss $\mathrm{CIV}_{\mathrm{S} 0}$ troughs as an intermediate case). Specifically, we consider BAL-trough profile properties (e.g., depth, width, EW, velocity, and profile shape) and BALvariability characteristics (e.g., EW and fractional EW variation strengths, depth variation profiles). Our comparative assessment points are the following:

1. The column density of outflowing gas is considerably larger along $\mathrm{LOS}_{\mathrm{SA}}$ than along $\mathrm{LOS}_{00}$, while the line-of-sight covering factors need not differ substantially. If the column density plays a role in setting trough depth, it is expected that $\mathrm{C}_{\mathrm{IV}} \mathrm{SA}$ troughs will generally be deeper than $\mathrm{C} \mathrm{IV}_{00}$ troughs (observational findings 1 and 2 above). While some BAL quasars are known to have highly saturated C IV troughs with depths largely set by the line-of-sight covering factor (rather than column density; see Section [1), it is not clear that all $\mathrm{C}$ IV troughs are highly saturated. Indeed, variability studies suggest that some C IV troughs are not highly saturated (see Section 1). The C IV troughs with detailed previous studies showing strong sat-

\footnotetext{
18 We appreciate that such correlated changes will have scatter owing to, e.g., time-dependent phenomena leading to local inhomogeneities (see Figure 3 of Proga et al. 2000). Overcoming such scatter is a prime driver for our utilization of large samples in this study.

19 The evidence for high global covering factors of low-ionization gas has been most notably presented for BAL quasars with detected Mg II absorption (e.g., Boroson \& Mevers 1992; Turnshek et al. 1994; Zhang et al. 2010). Although we primarily use Al III absorption to identify lines-of-sight with low-ionization gas, $\approx 75 \%$ of the objects in our $\mathrm{CIV}_{\mathrm{SA}}$ sample show Mg II absorption at corresponding velocities (see Section 2).
}

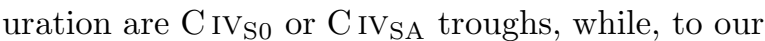
knowledge, no $\mathrm{CIV}_{00}$ troughs have been demonstrated to be highly saturated. Broadly consistent with this, we note that $\mathrm{P}_{\mathrm{V}}$ absorption corresponding to $\mathrm{CIV}_{00}$ troughs is rare and weak (see Section 3.3).

2. Given the poloidal velocity field of the model, we expect $\mathrm{C}_{\mathrm{IV}_{00}}$ troughs to have generally higher minimum outflow velocities than $\mathrm{CIV}_{\mathrm{SA}}$ troughs, as observed (see observational finding [3). This is because $\mathrm{LOS}_{00}$ intersects gas with a high outflow velocity without intersecting much gas with a low outflow velocity. $\mathrm{LOS}_{\mathrm{SA}}$, on the other hand, primarily intersects gas with a low outflow velocity. $\mathrm{LOS}_{\mathrm{SA}}$ might also intersect gas with a high outflow velocity if such gas extends close to the accretiondisk surface (i.e., prompt acceleration) at small radii, as appears required by our results showing high $v_{\max }$ values for $\mathrm{CIV}_{\mathrm{SA}}$ troughs. These same considerations can also explain the larger velocity widths of $\mathrm{CIV}_{\mathrm{SA}}$ troughs compared to $\mathrm{CIV}_{00}$ troughs (see observational finding 2). Figure 16 (lower panel) shows that the velocities for $\mathrm{LOS}_{00}$ appear to be $5000-10000 \mathrm{~km} \mathrm{~s}^{-1}$ along essentially the entire line-of-sight, and this is comparable to our measured $v_{\min }$ values for $\mathrm{CIV}_{00}$ troughs.

3. Given that the EW of a trough is set by a combination of its depth and width, we expect from the two comparative assessment points above that $\mathrm{CIV}_{\mathrm{SA}}$ troughs will have larger EWs than $\mathrm{CIV}_{00}$ troughs, as observed (see observational findings 1 and 2).

4. From the model we expect that Al III BALs will be mainly formed in the region close to the disk with high density and small poloidal velocity, while C IV BALs will be formed within both high-velocity and low-velocity gas. Thus, we expect that Al III troughs will reside within the lower velocity portions of C IV troughs, as observed (observational finding 1 and Voit et al. 1993).

5. The model shows that the optical depth is velocitydependent, and that it is generally higher for low poloidal velocities. Therefore, the low-velocity portions of $\mathrm{C}_{\mathrm{IV}_{\mathrm{SA}}}$ troughs that align with corresponding $\mathrm{Al}$ III troughs are likely to be more saturated than the high-velocity portions, perhaps partly leading to their larger depths (observational finding 1). Therefore these portions should be less variable than the high-velocity portions. This behavior is observed (observational finding 4).

6. Previous studies have presented evidence that ionization-level changes likely have a significant role in driving some BAL variability (see Section 1). Strongly saturated lines will be less susceptible to variability driven by ionization-level changes. Given that $\mathrm{CIV}_{\mathrm{SA}}$ troughs are likely more saturated than ${\mathrm{C} I V_{00}}_{\text {troughs, }}$ they are expected to be less variable. This is observed in an absolute sense and even more strongly in a fractional sense (observational findings 4 (6). 

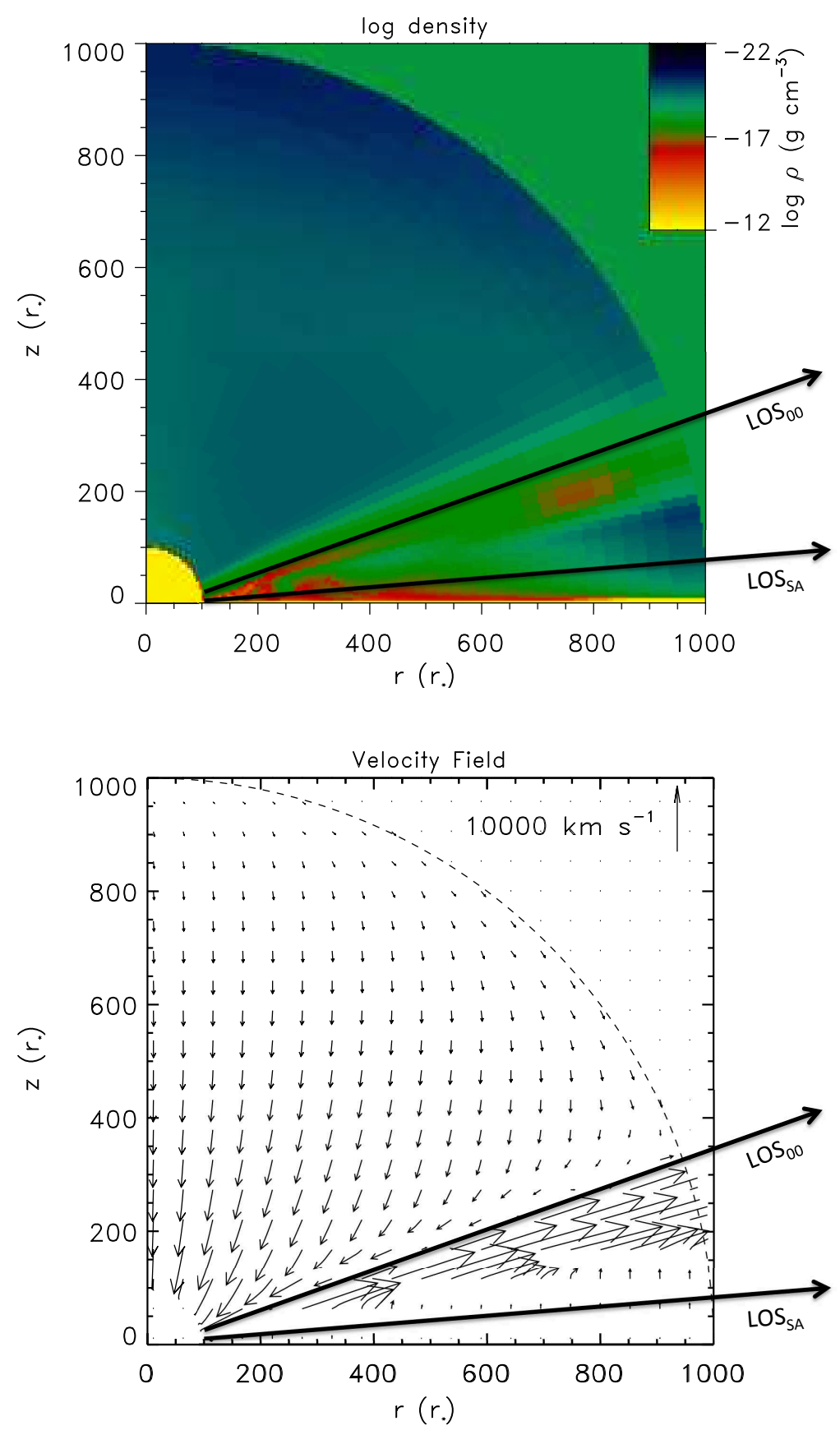

Figure 16. Density (top) and poloidal velocity (bottom) maps of the disk-wind model (adapted from Figure 2 of Proga et al. 2000). The SMBH is located at $(0,0)$. The $x$ and $y$-axes show the distance from the SMBH in units of the radius at the inner edge of the disk, where $r_{*}=3 \times r_{S}$ and $r_{S}$ is the Schwarzschild radius of a black hole. The bold black arrows indicate lines-of-sight with different viewing inclinations; $\mathrm{LOS}_{00}$ and $\mathrm{LOS}_{\mathrm{SA}}$ are the two lines-of-sight along which an observer would see $\mathrm{C}_{\mathrm{IV}} 00$ and $\mathrm{C}_{\mathrm{IV}} \mathrm{SA}$ troughs, respectively. 
7. The model indicates that the CIV optical depth along $\mathrm{LOS}_{\mathrm{SA}}$ is substantially larger than the Al III optical depth. Therefore, Al III troughs are expected to be more variable than $\mathrm{CIV}_{\mathrm{SA}}$ troughs, as they should be less saturated. This behavior is observed in an absolute sense and even more strongly in a fractional sense (observational finding 8 ).

The basic expectations of the disk-wind model for the characteristics of the $\mathrm{CIV}_{00}$ and $\mathrm{CIV}_{\mathrm{SA}}$ samples show qualitative agreement with our observational findings. In this model, a line-of-sight along which an observer would see a $\mathrm{CIV}_{\mathrm{SO}}$ trough is expected to intercept at least some less ionized gas than $\mathrm{LOS}_{00}$. Consistent with this expectation, our observational findings show that the $\mathrm{CIV}_{\mathrm{S} 0}$ sample is an intermediate case between the $\mathrm{CIV}_{00}$ and C IV SA samples (observational findings 1-7). 20

There are a number of ways the results above might be advanced, and here we highlight four that appear particularly promising. First, for the reasons discussed in Section 1, our current work has made use of the strong C IV, $\mathrm{Si}$ IV, and Al III BAL transitions as a basic measure of average line-of-sight ionization level. These transitions, spanning a factor of $\approx 2.5$ in ionization potential, have served effectively for our work. However, this ionizationpotential range could be expanded with the use of additional lower ionization (e.g., MgII, Fe II, and FeIII) and higher ionization (e.g., Ne VII, N v, O vI) transitions, thereby presumably probing wind zones even closer to and further from, respectively, the accretion disk. Second, the profiles and variability of the emission lines for large samples of BAL quasars with $\mathrm{C} \mathrm{IV}_{00}, \mathrm{C}_{\mathrm{IV}} \mathrm{S}$, and $\mathrm{C}_{\text {IV }}$ SA troughs should be measured systematically and compared with predictions (e.g., Murray \& Chiang 1997; Flohic et al. 2012). For a flattened Broad Line Region geometry, to first order one might expect the emission lines for BAL quasars with $\mathrm{C}_{\text {IV }}$ S troughs to be generally the broadest (though different emission lines, tracing different phases of the Broad Line Region, may behave differently). Third, the ongoing BOSS ancillary project and upcoming SDSS-IV Time Domain Spectroscopic Survey (TDSS 21 observations will both enlarge the sample size and improve the temporal sampling pattern for $\mathrm{BAL}$ quasars. This will allow variability to be used even more effectively as a tool for assessing correlated changes of ionization level, kinematics, and column density. Finally, while we have found generally good qualitative agreement with expectations for the disk-wind model, our ability to perform quantitative comparisons has been limited by the available simulation results. Future simulations capable of predicting trough profiles and variability (e.g., Higginbottom et al. 2013; D. Proga 2013, priv. comm.) can be quantitatively tested and constrained using large-sample measurements such as those provided here. Alternatives to the disk-wind model should also be developed to the point where quantitative testing is possible.

\footnotetext{
${ }^{20}$ We have also performed basic testing with the $\mathrm{CIV}_{\mathrm{s} 0}$, $\mathrm{CIV}_{\mathrm{sa}}$, and $\mathrm{CIV}_{\mathrm{Sa}}$ samples described in Section 3.1 and we generally find them to show suitably intermediate properties as well.

21 The current planning for SDSS-IV is briefly described at http://www.sdss3.org/future/
}

We gratefully acknowledge financial support from $\mathrm{Na}$ tional Science Foundation grant AST-1108604 (N.F.A., W.N.B., D.P.S.) and from NSERC (P.B.H.). We thank N. Arav and M. Eracleous for helpful discussions. We thank D. Proga for allowing us to adapt Figure 2 of Proga et al. (2000) as our Figure 16, We also thank the anonymous referee for constructive feedback.

Funding for SDSS-III has been provided by the Alfred P. Sloan Foundation, the Participating Institutions, the National Science Foundation, and the U.S. Department of Energy Office of Science. The SDSS-III web site is http://www.sdss3.org/

SDSS-III is managed by the Astrophysical Research Consortium for the Participating Institutions of the SDSS-III Collaboration including the University of Arizona, the Brazilian Participation Group, Brookhaven National Laboratory, Carnegie Mellon University, University of Florida, the French Participation Group, the German Participation Group, Harvard University, the Instituto de Astrofisica de Canarias, the Michigan State/Notre Dame/JINA Participation Group, Johns Hopkins University, Lawrence Berkeley National Laboratory, Max Planck Institute for Astrophysics, Max Planck Institute for Extraterrestrial Physics, New Mexico State University, New York University, Ohio State University, Pennsylvania State University, University of Portsmouth, Princeton University, the Spanish Participation Group, University of Tokyo, University of Utah, Vanderbilt University, University of Virginia, University of Washington, and Yale University.

\section{REFERENCES}

Allen, J. T., Hewett, P. C., Maddox, N., Richards, G. T., \& Belokurov, V. 2011, MNRAS, 410, 860

Anderson, L., Aubourg, E., Bailey, S., et al. 2012, MNRAS, 427, 3435

Arav, N. 1997, in Astronomical Society of the Pacific Conference Series, Vol. 128, Mass Ejection from Active Galactic Nuclei, ed. N. Arav, I. Shlosman, \& R. J. Weymann, 208

Arav, N., Becker, R. H., Laurent-Muehleisen, S. A., et al. 1999b, ApJ, 524, 566

Arav, N., Borguet, B., Chamberlain, C., Edmonds, D., \& Danforth, C. 2013, MNRAS, 436, 3286

Arav, N., Korista, K. T., de Kool, M., Junkkarinen, V. T., \& Begelman, M. C. 1999a, ApJ, 516, 27

Arav, N., de Kool, M., Korista, K. T., et al. 2001, ApJ, 561, 118

Becker, R. H., White, R. L., Gregg, M. D., et al. 2000, ApJ, 538, 72

Becker, R. H., White, R. L., \& Helfand, D. J. 1995, ApJ, 450, 559

Bolton, A. S., Schlegel, D. J., Aubourg, É., et al. 2012, AJ, 144, 144

Borguet, B. C. J., Edmonds, D., Arav, N., Benn, C., \& Chamberlain, C. 2012, ApJ, 758, 69

Boroson, T. A., \& Meyers, K. A. 1992, ApJ, 397, 442

Brotherton, M. S., van Breugel, W., Smith, R. J., et al. 1998, ApJ, 505, L7

Capellupo, D. M., Hamann, F., Shields, J. C., Rodríguez Hidalgo, P., \& Barlow, T. A. 2011, MNRAS, 413, 908

-. 2012, MNRAS, 422, 3249

Dawson, K. S., Schlegel, D. J., Ahn, C. P., et al. 2013, AJ, 145, 10

DiPompeo, M. A., Brotherton, M. S., \& De Breuck, C. 2012, ApJ, 752, 6

Eisenstein, D. J., Weinberg, D. H., Agol, E., et al. 2011, AJ, 142, 72

Faucher-Giguère, C.-A., Quataert, E., \& Murray, N. 2012, MNRAS, 420, 1347

Filiz Ak, N., Brandt, W. N., Hall, P. B., et al. 2012, ApJ, 757, 114 —. 2013, ApJ, 777, 168 
Gibson, R. R., Brandt, W. N., Gallagher, S. C., Hewett, P. C., \& Schneider, D. P. 2010, ApJ, 713, 220

Gibson, R. R., Brandt, W. N., Schneider, D. P., \& Gallagher, S. C. 2008, ApJ, 675, 985

Gibson, R. R., Jiang, L., Brandt, W. N., et al. 2009, ApJ, 692, 758

Gunn, J. E., Carr, M., Rockosi, C., et al. 1998, AJ, 116, 3040

Gunn, J. E., Siegmund, W. A., Mannery, E. J., et al. 2006, AJ, 131,2332

Hall, P. B., Anderson, S. F., Strauss, M. A., et al. 2002, ApJS, 141,267

Hamann, F. 1998, ApJ, 500, 798

Hewett, P. C., \& Wild, V. 2010, MNRAS, 405, 2302

Higginbottom, N., Knigge, C., Long, K. S., Sim, S. A., \& Matthews, J. H. 2013, MNRAS, 436, 1390

Kaspi, S., Brandt, W. N., George, I. M., et al. 2002, ApJ, 574, 643

Kelly, B. C. 2007, ApJ, 665, 1489

Latta, R. B., 1981, J. Am. Stat. Assoc., 76, 713

Lavalley, M., Isobe, T., \& Feigelson, E. 1992, Astronomical Data Analysis Software and Systems I, 25, 245

Leighly, K. M., Hamann, F., Casebeer, D. A., \& Grupe, D. 2009, ApJ, 701, 176

Lucy, A. B., Leighly, K. M., Terndrup, D. M., Dietrich, M., \& Gallagher, S. C. 2014, ArXiv e-prints, arXiv:1401.0605

Lundgren, B. F., Wilhite, B. C., Brunner, R. J., et al. 2007, ApJ, 656,73

Murray, N., Chiang, J., Grossman, S. A., \& Voit, G. M. 1995, ApJ, 451, 498

Ogle, P. M., Cohen, M. H., Miller, J. S., et al. 1999, ApJS, 125, 1
Pei, Y. C. 1992, ApJ, 395, 130

Press, W. H., Teukolsky, S. A., Vetterling, W. T., \& Flannery, B. P. 2007, Numerical recipes. The art of scientific computing (Cambridge University Press)

Proga, D., Stone, J. M., \& Kallman, T. R. 2000, ApJ, 543, 686 Reichard, T. A., Richards, G. T., Hall, P. B., et al. 2003, AJ, 126, 2594

Ross, N. P., Myers, A. D., Sheldon, E. S., et al. 2012, ApJS, 199, 3 Schlegel, D. J., Finkbeiner, D. P., \& Davis, M. 1998, ApJ, 500, 525

Shen, Y., Richards, G. T., Strauss, M. A., et al. 2011, ApJS, 194, 45

Smee, S. A., Gunn, J. E., Uomoto, A., et al. 2013, AJ, 146, 32

Spergel, D. N., Verde, L., Peiris, H. V., et al. 2003, ApJS, 148, 175

Trump, J. R., Hall, P. B., Reichard, T. A., et al. 2006, ApJS, 165, 1

Turnshek, D. A., Espey, B. R., Kopko, Jr., M., et al. 1994, ApJ, 428, 93

Vivek, M., Srianand, R., Petitjean, P., et al. 2012, MNRAS, 423, 2879

Voit, G. M., Weymann, R. J., \& Korista, K. T. 1993, ApJ, 413, 95

Weymann, R. J., Morris, S. L., Foltz, C. B., \& Hewett, P. C. 1991, ApJ, 373, 23

Wildy, C., Goad, M. R., \& Allen, J. T. 2013, MNRAS, arXiv:1310.5674

York, D. G., Adelman, J., Anderson, Jr., J. E., et al. 2000, AJ, 120,1579

Zhang, S., Wang, T.-G., Wang, H., et al. 2010, ApJ, 714, 367 